\title{
A versatile approach to novel homo-C-nucleosides based on aldehydes and acetylenic ketones derived from ribo- and 2-deoxy- ribofuranose $\mathrm{C}$-glycosides
}

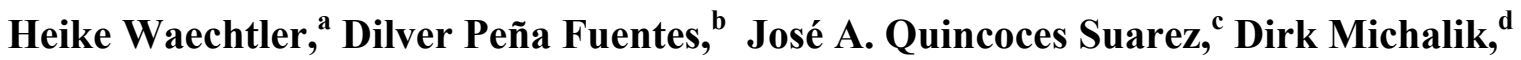 \\ Alexander Villinger, ${ }^{\mathrm{b}}$ Udo Kragl, ${ }^{\mathrm{b}}$ and Christian Vogel $^{\mathrm{b}}$ * \\ ${ }^{a}$ Agilent Technologies, Hewlett-Packard-Str. 8, D-76337 Waldbronn, Germany \\ ${ }^{b}$ University of Rostock, Institute of Chemistry, Albert-Einstein-Str. 3a. D-18059 Rostock, \\ Germany \\ ${ }^{c}$ Laboratory of Organic Synthesis, Universidade Bandeirante de São Paulo, São Paulo, Brazil \\ ${ }^{d}$ Leibniz Institute for Catalysis at the University of Rostock, Albert-Einstein-Str. 29a, D-18059 \\ Rostock, Germany \\ E-mail: christian.vogel@uni-rostock.de
}

\section{Dedicated to Prof. Dr. Rainer Beckert on the Occasion of his $60^{\text {th }}$ Birthday}

\begin{abstract}
A series of ribofuranosyl- and 2-deoxyribofuranosyl homo- and spacered-C-nucleosides have been synthesized by reaction of fully protected 3-(1-deoxy- $\beta$-D-ribofuranosyl-1-yl)propanal (1), 3-(1,2-dideoxy- $\beta$-D-ribofuranos-1-yl)propanal (14), 1-(1-desoxy- $\beta$-D-ribofuranos-1-yl)pent-4-yn3-on (19), 1-(1-desoxy- $\beta$-D-ribofuranos-1-yl)-5-phenyl-pent-4-yn-3-on (20), 1-(1,2-didesoxy- $\beta$ D-ribofuranos-1-yl)pent-4-yn-3-on (29), and 1-(1,2-didesoxy- $\beta$-D-ribofuranos-1-yl)-5-phenylpent-4-yn-3-on (30) with different nucleophiles. The preparation of $\mathbf{1}$ and $\mathbf{1 4}$ proceeds by Knoevenagel reaction with malononitrile, cyanoacetamide and 2-cyano- $N$-(4-methoxyphenyl)acetamide and subsequent cyclization with sulphur to thiophenes 5, 7, 8, 16 and then by cyclization with triethyl orthoformate to give thienopyrimidine 6 and thienopyrimidinone 9, 10, and 17. Treatment of acetylenic ketones 19, 20, 29, and $\mathbf{3 0}$ with acetamidinium chloride, benzamidinium chloride, and $S$-methylisothiouronium sulphate provided the corresponding pyrimidines 21-26, 31, 32. Finally, the use of 4H-1,2,4-triazol-3-amine and 2-aminobenzimidazole as 1,3-N,N'-dinucleophiles afforded the triazolopyrimidines 35, 39 and the pyrimidobenzimidazoles 36, 37, and 40, respectively. Deprotection of a selected number of Cnucleosides was achieved by one or two steps procedure without serious problems. That makes these C-nucleosides promising candidates for the synthesis of monomers suitable for solid phase nucleic acid oligomerization.
\end{abstract}

Keywords: Ribose, 2-deoxy-ribose, C-nucleosides, thiophenes, pyrimidines, thienopyrimidines 


\section{Introduction}

The interest in nucleoside analogues is unbroken and their design and synthesis have been done with quite different intentions e.g. synthesis of homo-C-nucleosides with potential biological activity, ${ }^{1-3}$ tools for elucidating the structural and functional properties of damaged DNA, ${ }^{4}$ illustration of hybridizations and conformational changes of DNA and RNAs, ${ }^{5}$ and to give an answer to the question why DNA evolved on Earth to have the structure that it does. ${ }^{6-10}$

Pursuing a program directed at the synthesis of homo-C-nucleosides, we have described previously an efficient route for the preparation of $\beta$-allyl C-glycosides of D-ribofuranose and 2deoxy-D-ribofuranose. ${ }^{11}$ Recently we have reported the synthesis of consecutive compounds e.g. alcohols, amines, aldehydes, and acetylenic ketones. ${ }^{12}$ In this contribution we present our results on transformation of the furnished aldehydes $(\mathbf{1 , 1 4})$ and acetylenic ketones $(\mathbf{1 9}, \mathbf{2 0}, \mathbf{2 9}, \mathbf{3 0})$ as versatile intermediates into a selected number of different heterocycles to make the synthetic potential of these precursors visible.

\section{Results and Discussion}

\section{Synthesis of thienopyrimidine homo-C-nucleosides}

The synthesis started from the aldehyde $\mathbf{1}$ which was readily obtainable from $\beta$-allyl C-glycoside of D-ribose ${ }^{11}$ via hydroboration/ oxidation and selective oxidation of the corresponding alcohol. ${ }^{12}$ Treatment of 1 with malononitrile, cyanoacetamide and 2-cyano- $N$-(4-methoxyphenyl)acetamide provided the corresponding Knoevenagel products $2-4$ in $60 \%, 51 \%$, and $77 \%$, respectively (Scheme 1). The reaction was carried out by using an excess of the $\mathrm{CH}$-acidic compounds and basic aluminium oxide in boiling toluene. ${ }^{13}$ The reaction time varied between 2 and $16 \mathrm{~h}$ as monitored by TLC. All analytical data were in accordance with the proposed structures. Additionally, the values for the coupling constants $J_{\mathrm{H} 2-\mathrm{CN}}$ and $J_{\mathrm{H} 2-\mathrm{C}=\mathrm{O}}(13-14 \mathrm{~Hz}$ and 5-6 Hz, respectively) determined from coupled ${ }^{13} \mathrm{C}$ NMR spectra confirmed the $E$-configuration of structures 3 and 4 . When the compounds 2-4 were treated with elemental sulphur and triethylamine in $N, N$-dimethylformamide (DMF) $)^{13,14}$ for $2 \mathrm{~h}$ at r.t. the light yellow aminothiophenes 5, 7, and $\mathbf{8}$ were obtained in about $80 \%$ yield. The ${ }^{1} \mathrm{H}$ NMR spectra showed signals at $\delta 2.81-3.02$ and the ${ }^{13} \mathrm{C}$ NMR spectra provided signals at $\delta 33.7-33.9$ characteristic of the methylene unit of homo-C-nucleosides. For the synthesis of 4-aminothienopyrimidine 6 compound 5 was reacted with triethyl orthoformate under reflux for $2 \mathrm{~h}$. Without purification the resulting formimidate was treated with a saturated ethanolic ammonia solution to afford the desired compound $\mathbf{6}$ in $74 \%$ overall yield. ${ }^{15}$ Cyclization to the thienopyrimidinones 9 and $\mathbf{1 0}$ were achieved when a mixture of compounds $\mathbf{7}$ or $\mathbf{8}$ and triethyl orthoformate were heated at reflux in DMF for 7-10 h. In spite of the drastic reaction conditions the desired derivatives 9 and 10 were obtained in about $70 \%$ yield. In the ${ }^{1} \mathrm{H}$ NMR spectra singlets were observed at $\delta 8.05$ and 8.02 characteristic of $\mathrm{H}-2$ of $\mathbf{9}$ and $\mathbf{1 0}$, respectively. 


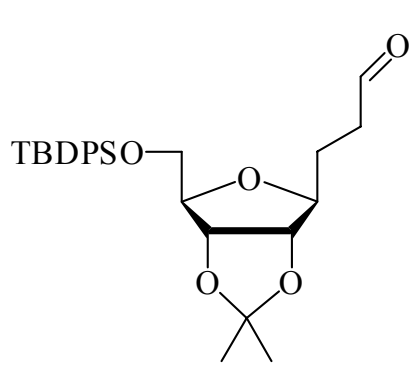

1

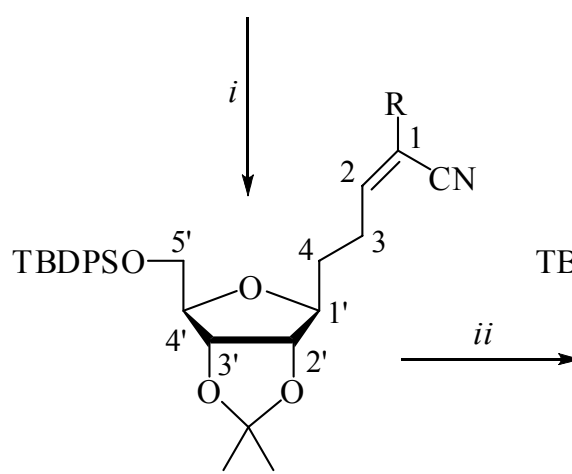

$2 \mathrm{R}=\mathrm{CN}$

$3 \mathrm{R}=\mathrm{CONH}_{2}$

$4 \mathrm{R}=\mathrm{CONHC}_{6} \mathrm{H}_{4} \mathrm{OMe}-p$

$7 \mathrm{R}=\mathrm{H}($ from 3$)$

$8 \mathrm{R}=\mathrm{C}_{6} \mathrm{H}_{4} \mathrm{OMe}-p$ (from 4 )

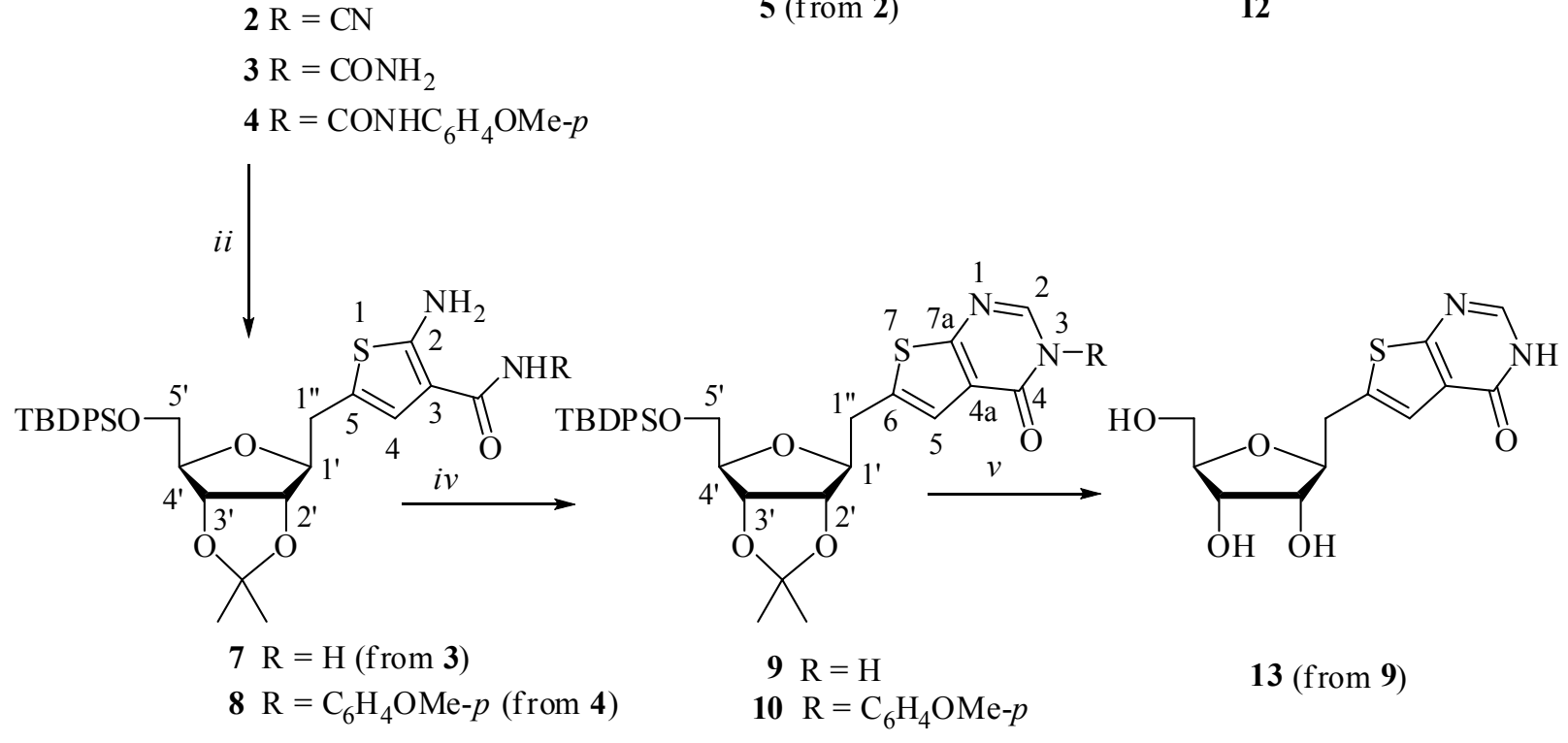

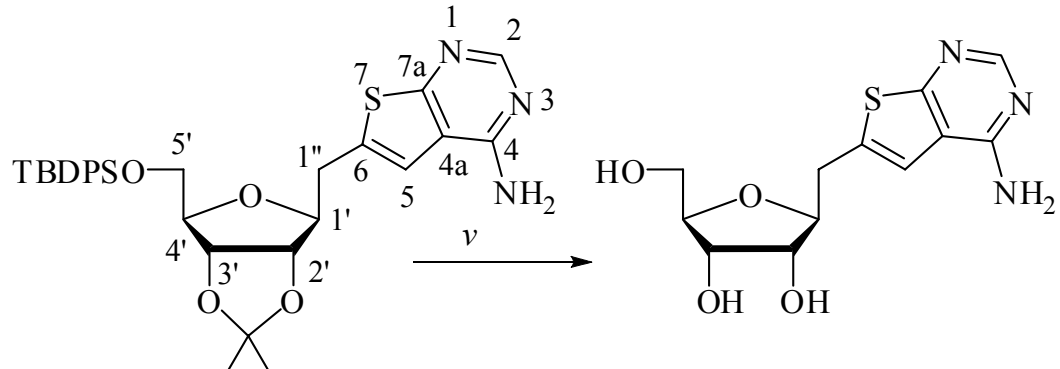

11

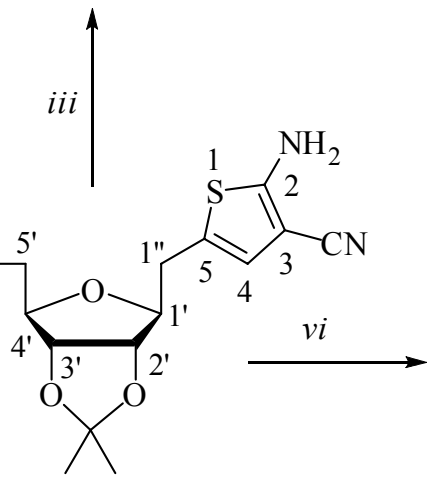

5 (from 2)

12

Scheme 1. Syntheses of thiophenes 5, 7, 8, pyrimidine 6, and pyrimidinone 17 via Knoevenagel products 2-4. Reagents and conditions: (i) CH-acid compounds, $\mathrm{Al}_{2} \mathrm{O}_{3}$, dry toluene, reflux, 2-16 h; (ii) sulfur, $\mathrm{NEt}_{3}$, DMF, r.t. $2 \mathrm{~h}$; (iii) $\mathrm{HC}(\mathrm{OEt})_{3}$, reflux 2h, then ethanol-ammonia, reflux $2 \mathrm{~h}$; (iv) $\mathrm{HC}(\mathrm{OEt})_{3}$, dry DMF, reflux, 7-10 h; (v) $90 \%$ aq $\mathrm{CF}_{3} \mathrm{CO}_{2} \mathrm{H}, \mathrm{CH}_{2} \mathrm{Cl}_{2}$, r.t.; (vi) $\mathrm{Bu} 4 \mathrm{NF}$, 1,4dioxane, r.t. 5 h, then $90 \%$ aq $\mathrm{CF}_{3} \mathrm{CO}_{2} \mathrm{H}, \mathrm{CH}_{2} \mathrm{Cl}_{2}$, r.t. 
Finally, treatment of 9 and 6 with $90 \%$ trifluoroacetic acid in $\mathrm{CH}_{2} \mathrm{Cl}_{2}$ removed both the silyl and isopropylidene protecting groups, providing the unprotected derivatives $\mathbf{1 1}$ and $\mathbf{1 3}$ in $90 \%$ yield. In contrast, deprotection of thiophene 5 required a two step procedure. Firstly, the tertbutyldiphenylsilyl group (TBDPS) was cleaved off by treatment of $\mathbf{5}$ with a solution of tetrabutylammonium fluoride (TBAF) in 1,4-dioxane. After $5 \mathrm{~h}$, the isopropylidene group was then removed with $90 \%$ trifluoroacetic acid in $\mathrm{CH}_{2} \mathrm{Cl}_{2}$ to give 12 in $87 \%$ overall yield.

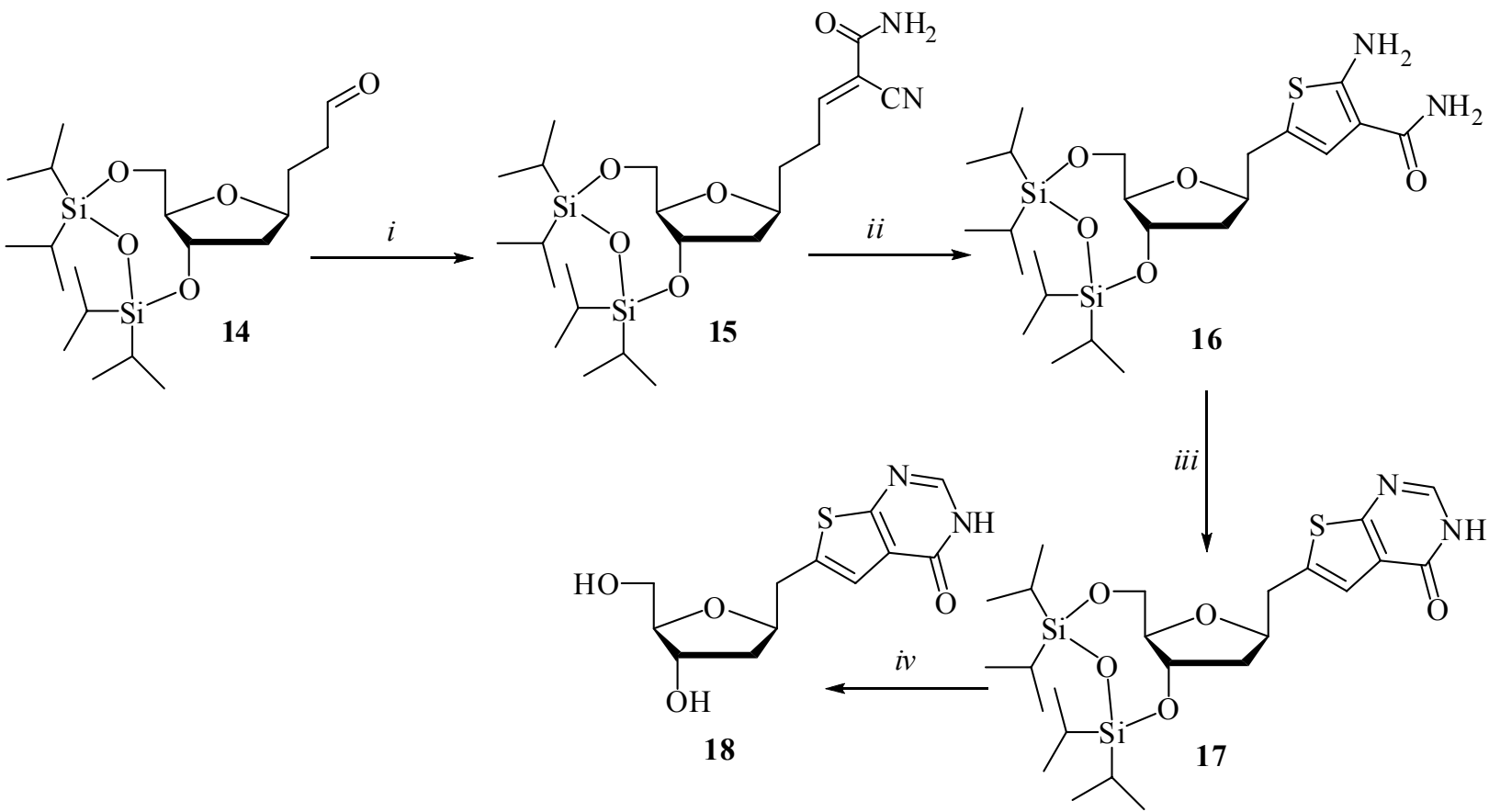

Scheme 2. Synthesis of thiophenecarboxamide 16 and pyrimidinon 17. Reagents and conditions: (i) 2-cyanoacetamide, $\mathrm{Al}_{2} \mathrm{O}_{3}$, dry toluene, reflux, $24 \mathrm{~h}$; (ii) sulfur, $\mathrm{NEt}_{3}$, DMF, r.t. 2 h; (iii) $\mathrm{HC}(\mathrm{OEt})_{3}$, dry DMF, reflux, 10 h; (iv) $\mathrm{Bu}_{4} \mathrm{NF}$, 1,4-dioxane, r.t. 2 h.

In a previous paper, ${ }^{11}$ we described an efficient route to transfer $\beta$-allyl C-glycoside of Dribose into the corresponding 2-deoxy ribofuranose. Employment of exactly the same conditions of hydroboration-oxidation and consecutive selective oxidation of the corresponding alcohol furnished aldehyde 14. ${ }^{12}$ The versatility of 14 was demonstrated by the preparation of thieonopyrimidinone $\mathbf{1 8}$ by the same sequence of reaction steps as described for compound $\mathbf{1 3}$ (Scheme 2). Even the reaction conditions were identical only slightly differences of reaction time and yield occur. Thus, Knoevenagel product 15 was obtained in 52\% yield. Transformation of 15 into thiophene 16 (73\%) was followed by ring closure reaction to provide 17 in $69 \%$ yield. Deprotection was simply achieved by treatment of $\mathbf{1 7}$ with TBAF in a solution of 1,4-dioxane. After $2 \mathrm{~h}$ at r.t. derivative $\mathbf{1 8}$ was obtained in $78 \%$ yield.

As described previously, ${ }^{12}$ aldehydes 1 and 14 can be converted to acetylenic ketones 19, 20 and 29, 30 by reaction with ethynylmagnesium bromide or phenylethynyllithium, respectively, 
and followed by oxidation of the diastereomeric alcohols. Analytical sample of $\mathbf{2 0}$ was obtained by crystallization from ethyl acetate- $n$-hexane. Its constitution was confirmed by X-ray diffraction studies (Figure 1).

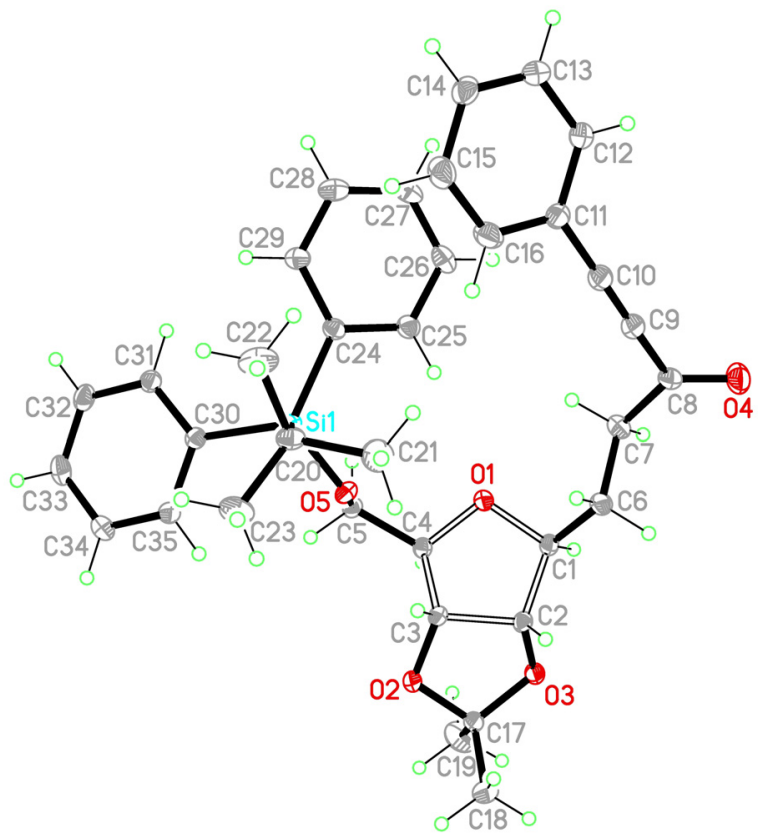

\section{Figure 1}

Molecular structure of alkynyl ketone 20 with atom labeling scheme. Thermal ellipsoids are drawn at the $30 \%$ probability level. Puckering parameters are $\mathrm{q} 2=0.3233(1)$ and $\Phi 2=-35.96(1)$ for the tetrahydrofuran ring.

\section{Synthesis of pyrimidine-spacered C-nucleosides}

Acetylenic ketones are a versatile class of compounds which can be used as starting materials for the synthesis of a broad variety of heterocycles. ${ }^{16-20}$ Herein we report efficient short synthesis of substituted pyrimidines and triazolopyrimidines. Following the strategy of Addlington et al, ${ }^{17}$ who reported the reaction of acetylenic ketones with amidinium salts using ethyl acetate/water as solvent and sodium carbonate as base, 19 and $\mathbf{2 0}$ were treated with acetamidinium chloride, benzamidinium chloride, and $S$-methylisothiouronium sulphate to give the corresponding pyrimidines 21-26 separated from the tetrahydrofuran ring by an ethylene group (Scheme 3).

All reactions proceeded in good (60\%) to excellent yield (quantitative). As expected, all analytical data were in agreement with the proposed structures. We examined the stepwise and complete deprotection of the obtained pyrimidines using the example of compound 25 . Treatment of 25 with aq $\mathrm{HCl}$ in $\mathrm{EtOH}$ resulted in simultaneous removal of both silyl and isopropylidene protecting groups to give $\mathbf{2 8}$ in $74 \%$ yield. On the other hand, in the presence of 
TBAF in 1,4-dioxane only the TBDPS group was removed and the partial protected derivative $\mathbf{2 7}$ was obtained in 85\% yield. Again, the 2-deoxy acetylenic ketones $\mathbf{2 9}$ and $\mathbf{3 0}$ were allowed to react with selected amidinium salts e.g. $S$-methylisothiouronium sulphate and acetamidinium chloride to provide the pyrimidines 31 and 32, respectively (Scheme 4). Deprotection of 31 with TBAF in 1,4-dioxane afforded 33 in $78 \%$ yield.

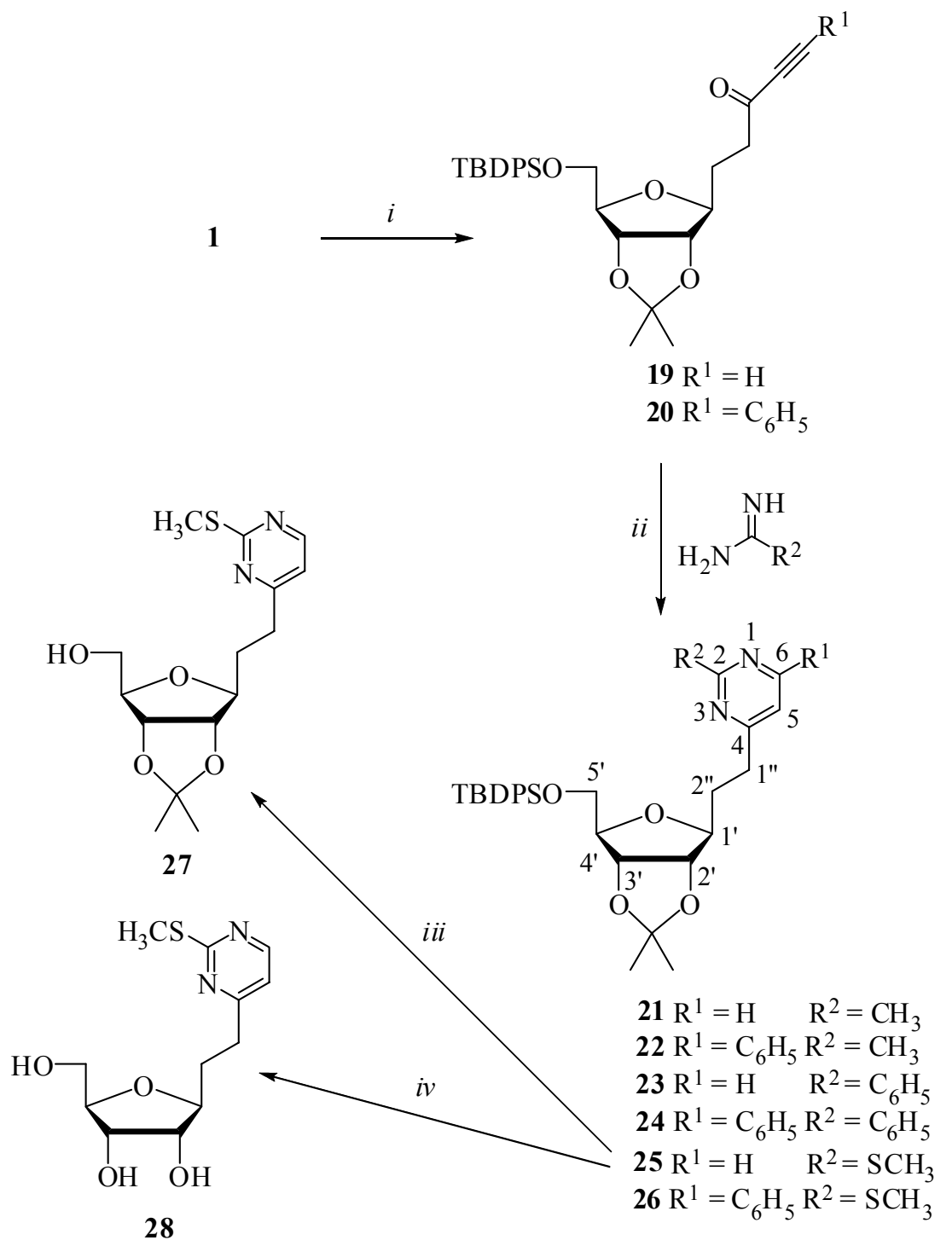

Scheme 3. Synthesis of pyrimidines 21-26 via acetylenic ketones 19 and 20. Reagents and conditions: $(i)$ ethynylmagnesium bromide or phenylethynyllithium, dry THF, r.t. $4 \mathrm{~h}$, then DessMartin oxidation; (ii) acetamidinium, benzamidinium or $S$-methylisothiouronium salts, cat. $\mathrm{H}_{2} \mathrm{O}$, $\mathrm{Na}_{2} \mathrm{CO}_{3}$, AcOEt, reflux, 2-24 h; (iii) $\mathrm{Bu}_{4} \mathrm{NF}$, 1,4-dioxane, r.t. 4 h; (iv) aq $\mathrm{HCl}$ (1M), EtOH, r.t. 12 $\mathrm{h}$. 


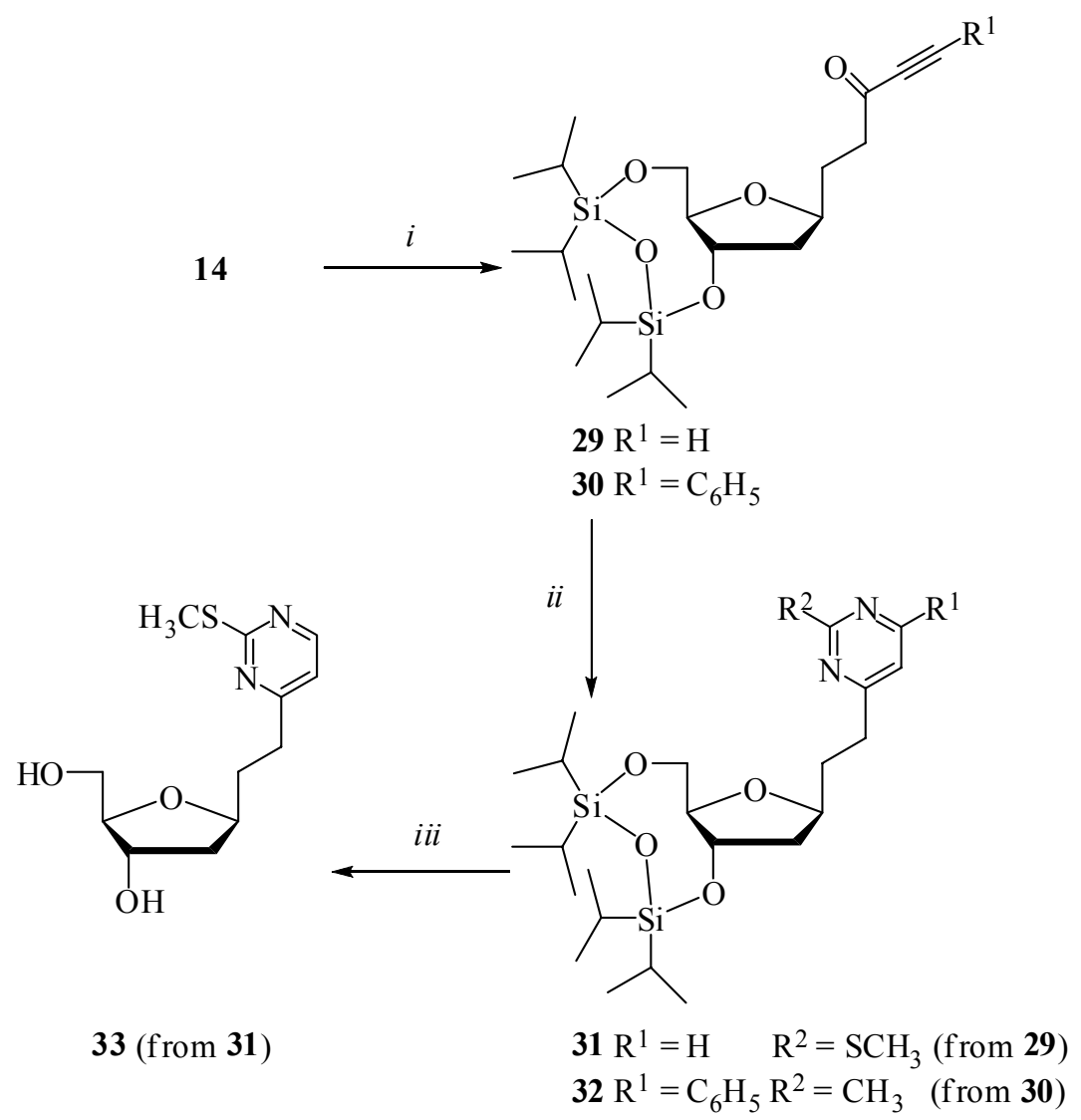

Scheme 4. Synthesis of pyrimidines 31 and $\mathbf{3 2}$ via acetylenic ketones 29 and $\mathbf{3 0}$. Reagents and conditions: (i) ethynylmagnesium bromide or phenylethynyllithium, dry THF, r.t. 4 h, then DessMartin oxidation; (ii) $S$-methylisothiouronium sulphate (for 31) and acetamidinium chloride (for 32), cat. $\mathrm{H}_{2} \mathrm{O}, \mathrm{Na}_{2} \mathrm{CO}_{3}$, AcOEt, reflux, 3-24 h; (iii) $\mathrm{Bu}_{4} \mathrm{NF}$, 1,4-dioxane, r.t. 24 h.

\section{Synthesis of triazolo- and pyrimidinobenzimidazole-spacered C-nucleosides}

Nucleophilic attack of 4H-1,2,4-triazol-3-amine on the triple bond of ynone 19 in boiling EtOH resulted in compound $\mathbf{3 4}$ in $89 \%$ yield (Scheme 5 ). ${ }^{21}$ The ${ }^{1} \mathrm{H}$ and ${ }^{13} \mathrm{C}$ NMR spectra of $\mathbf{3 4}$ were fully consistent with the assigned structure. As expected, no signals of acetylenic carbon atoms were observed in the ${ }^{13} \mathrm{C}$-NMR spectra, but a resonance was visible at $\delta 151.7$ for C-3"' of the triazole ring. The $E$-configuration of the addition product was evident from the large coupling constant ${ }^{3} J_{4,5} 13.3 \mathrm{~Hz}$ in the ${ }^{1} \mathrm{H}$ NMR spectra. In order to prepare a fused heterocycle enone $\mathbf{3 4}$ was treated with sodium ethanolate at r.t. for $1 \mathrm{~h}$ to afford triazolopyrimidine 35 in $62 \%$ yield. Analogous to that addition reaction 2-aminobenzimidazole was used as 1,3- $N, N$ '-dinucleophile and allowed to react with ynone $\mathbf{1 9}$ and $\mathbf{2 0}$. Suprisingly, the TLC of the reaction solution showed in each case the formation of a mixture two products after reflux $(\mathrm{EtOH})$ for $2 \mathrm{~h}$. The stepwise formation of compound $\mathbf{3 5}$ strongly suggested that here a mixture of an addition products (in parenthesis Scheme 5) and the fused heterocycles $\mathbf{3 6}$ and $\mathbf{3 7}$ were observed. 


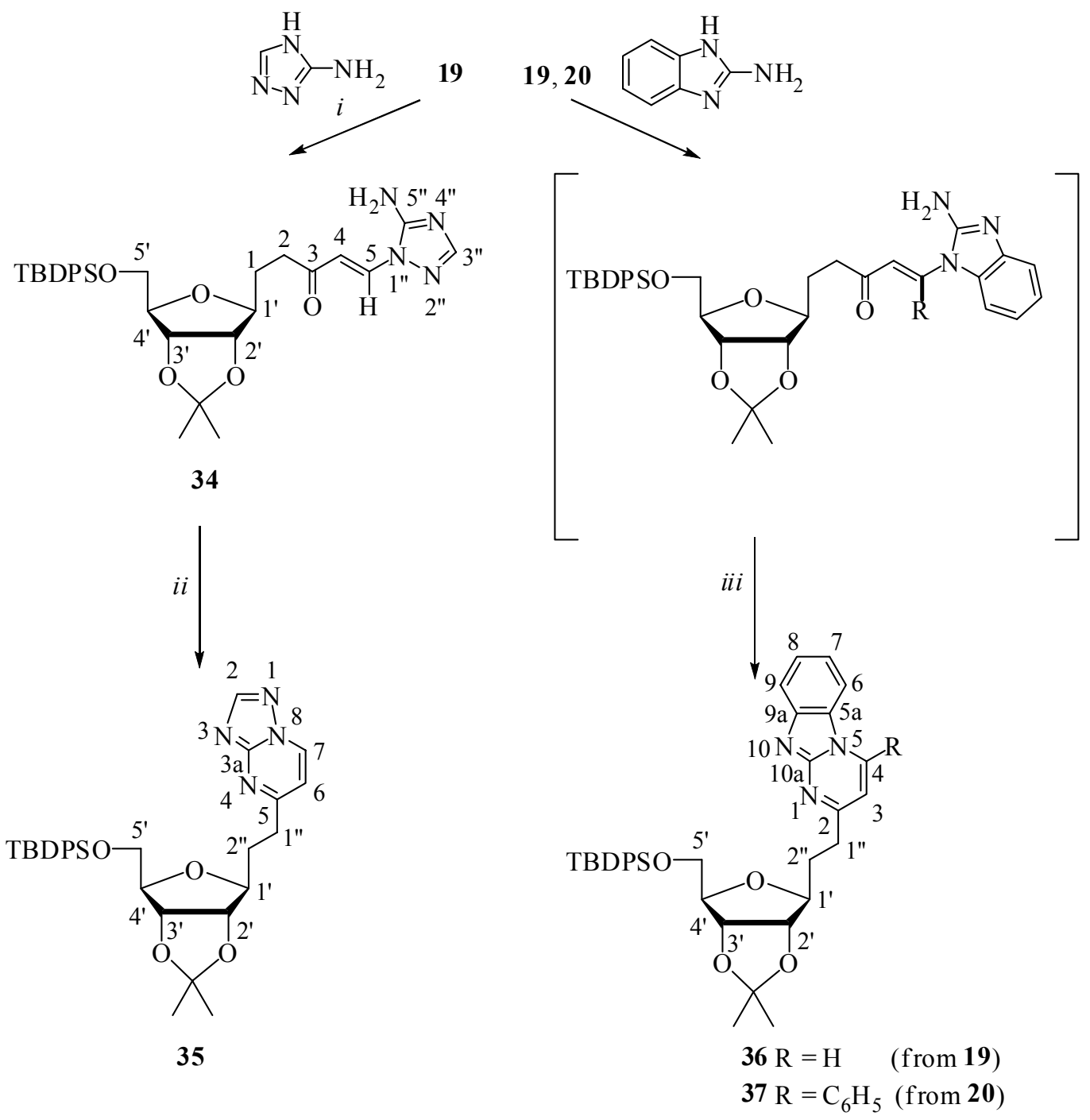

Scheme 5. Synthesis of triazolopyrimidine 35 and pyrimidobenzimidazoles $\mathbf{3 6}$ and $\mathbf{3 7}$. Reagents

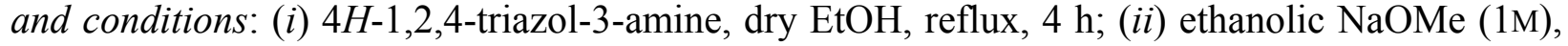
r.t., $1 \mathrm{~h}$; (iii) $1 H$-benzo[d]imidazole-2-amine, dry EtOH, reflux, $2 \mathrm{~h}$, then ethanolic $\mathrm{NaOMe} 1 \mathrm{M}$ ), r.t. $1 \mathrm{~h}$.

Indeed, treatment of the unseparated reaction mixture with sodium ethanolate at r.t. for $1 \mathrm{~h}$ caused disappearance of the side-products and provided $\mathbf{3 6}$ and 37 in $76 \%$ and $80 \%$ yield, respectively. The protocol of the formation of triazolopyrimidine and pyrimidobenzimidazole by reaction of 4H-1,2,4-triazole-3-amine and 2-aminobenzimidazole, respectively, was now transferred to the ynone 29 (Scheme 6). Fortunately, the course of all reactions was comparable to ynone 19. Consequently, triazolopyrimidine 39 and pyrimidobenzimidazole $\mathbf{4 0}$ were obtained in $69 \%$ and $63 \%$, respectively. The regioselectivity of the ring closure reactions was evident from NOESY and HMBC experiments. 


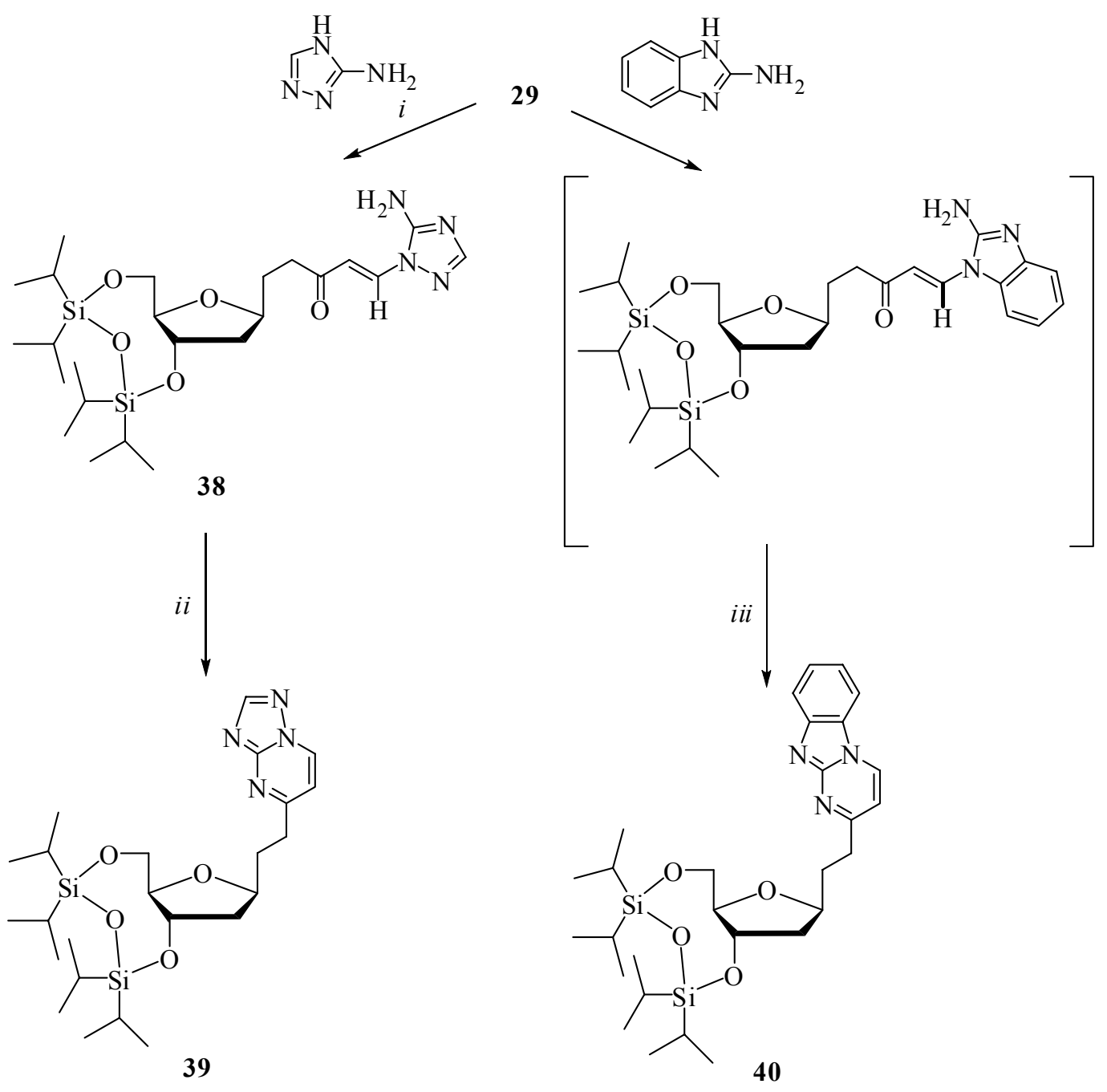

Scheme 6. Synthesis of triazolopyrimidine 39 and pyrimidobenzimidazole 40. Reagents and conditions: (i) 4H-1,2,4-triazol-3-amine, dry EtOH, reflux, 4 h; (ii) ethanolic $\mathrm{NaOMe}$ (1M), r.t., 1 h; (iii) $1 H$-benzo[d]imidazole-2-amine, dry EtOH, reflux, 2 h, then ethanolic $\mathrm{NaOMe} 1$ M), r.t. 1 h.

In summary, we have shown that fully protected ribofuranosylpropanal (1), 2-deoxyribofuranosylpropanal (14) and the corresponding alkynyl ketones 19, 20, 29, and 30 are suitable intermediates for the preparation of homo- and spacered C-nucleosides. The tetrahydrofuran ring and the protecting group pattern is stable enough even under drastic reaction conditions to allow the synthesis of a broad variety of heterocyclic systems. In one of our next papers, we will describe the conversion of some of our C-nucleosides into building blocks suitable for solid phase synthesis of nucleic acid oligomers. 


\section{Experimental Section}

General. Melting points were determined with a Boetius micro-heating plate BHMK 05 (Rapido, Dresden) and were not corrected. Optical rotation was measured for solutions in a 2-cm cell with an automatic polarimeter GYROMAT (Dr. Kernchen Co.). ${ }^{1} \mathrm{H}$ NMR spectra (250.13 and $300.13 \mathrm{MHz})$ and ${ }^{13} \mathrm{C}$ NMR spectra $(62.9$ and $75.5 \mathrm{MHz})$ were recorded on Bruker spectrometers AVANCE 250 and AVANCE 300, respectively, at $298 \mathrm{~K}$. The chemical shifts are referenced to solvent signals $\left(\mathrm{CDCl}_{3}: \delta{ }^{1} \mathrm{H}=7.26, \delta{ }^{13} \mathrm{C}=77.0\right.$; DMSO- $d_{6}: \delta{ }^{1} \mathrm{H}=2.49, \delta{ }^{13} \mathrm{C}$ $=39.7 ; \mathrm{CD}_{3} \mathrm{OD}: \delta{ }^{1} \mathrm{H}=3.30, \delta{ }^{13} \mathrm{C}=49.3$ ). Signal assignment was performed by recording the DEPT spectra, in some cases also by recording of two-dimensional ${ }^{1} \mathrm{H},{ }_{1}^{1} \mathrm{H} \operatorname{COSY},{ }^{13} \mathrm{C},{ }^{1} \mathrm{H}$ HETCOR and ${ }^{1} \mathrm{H},{ }^{13} \mathrm{C}$ HMBC spectra. For NMR numbering of atoms see Scheme 1,3 and 5. Mass spectra were recorded on an AMD 402/3 spectrometer (AMD Intectra GmbH). Elemental analysis was performed on a CHNS-Flash-EA-1112 instrument (Thermoquest). For the X-ray structure determination of compound $\mathbf{2 0}$ an X8Apex system with CCD area detector was used $(\lambda$ $=0.71073 \AA$, graphite monochromator). The structures were solved by direct methods (BrukerSHELXTL). The refinement calculations were done by the full-matrix least-squares method of Bruker SHELXTL, Vers.5.10, Copyright 1997, Bruker Analytical X-ray Systems. All nonhydrogen atoms were refined anisotropically. The hydrogen atoms were put into idealised positions and refined using the riding models. Crystallographic data for the structure analysis has been deposited with the Cambridge Crystallographic Data Centre, CCDC No 827042 for compound 20. Copies of this information may be obtained free of charge from: The Director, CCDC, 12 Union Road Cambridge, CB2 1EZ UK, Fax. (int code) +44(1223)336-033 or via Email: deposit @ccdc. cam.ac.uk or www:http://www. ccdc. cam.ac.uk. All washing solutions were cooled to $\sim 5^{\circ} \mathrm{C}$. The $\mathrm{NaHCO}_{3}$ solution was saturated. Reactions were monitored by thinlayer chromatography (TLC, Silica Gel 60, $\mathrm{F}_{254}$, Merck KGaA). The followings solvent systems (v/v) were used: (A) ethyl acetate, $\left(\mathrm{B}_{1}\right)$ 2:1, $\left(\mathrm{B}_{2}\right)$ 1:1, $\left(\mathrm{B}_{3}\right)$ 2:3, $\left(\mathrm{B}_{4}\right)$ 1:2, $\left(\mathrm{B}_{5}\right)$ 1:3, $\left(\mathrm{B}_{6}\right)$ 1:4, $\left(\mathrm{B}_{7}\right)$ 1:5 ethyl acetate $-n$-hexane; $\left(\mathrm{C}_{1}\right)$ 1:1, $\left(\mathrm{C}_{2}\right)$ 4:1, $\left(\mathrm{C}_{3}\right)$ 5:1, $\left(\mathrm{C}_{4}\right)$ 6:1 ethyl acetate - methanol; (D) 12:1:0.1 ethyl acetate - methanol - acetic acid. The spots were made visible by dipping the TLC plates into a methanolic $10 \% \mathrm{H}_{2} \mathrm{SO}_{4}$ solution and charring with a heat gun for 3-5 min. Preparative flash chromatography was performed by elution from columns of slurry-packed Silica Gel 60 (Merck, 63-200 $\mu \mathrm{m}$ ). All solvents and reagents were purified and dried according to standard procedures. ${ }^{22}$ After classical work up of the reactions mixtures, the organic layer were dried over $\mathrm{MgSO}_{4}$, and then concentrated under reduced pressure (rotary evaporator).

\section{KNOEVENAGEL reaction with aldehyde (1). General procedure}

Malononitrile (377 mg, $5.7 \mathrm{mmol})$, 2-cyanoacetamide (479 $\mathrm{mg}, 5.7 \mathrm{mmol})$ or 2-cyano- $N$-(4methoxyphenyl)acetamide $(1.08$ g. $5.7 \mathrm{mmol})$ was added to a stirred solution of 3-(5-O-tertbutyldiphenylsilyl-2,3-O-isopropylidene-1-deoxy- $\beta$-D-ribofuranosyl-1-yl)propanal (1, $1.17 \mathrm{~g}, 2.5$ mmol) and aluminium oxide (653 mg, $6.4 \mathrm{mmol}$, basic activated 90, 101076 MERCK) in dry toluene $(75 \mathrm{~mL})$. After heating under reflux for $2-16 \mathrm{~h}$ (monitored by TLC), the reaction mixture 
was cooled to r.t., the insoluble solids were filtered off, and the filtrate was concentrated. The residue was purified by flash chromatography.

\section{2-[3-(5-O-tert-Butyldiphenylsilyl-2,3-O-isopropylidene-1-deoxy- $\beta$-D-ribofuranos-1-yl)pro-} pylidene]malononitrile (2). Reaction time $2.5 \mathrm{~h}$; flash chromatography solvent $\mathrm{B}_{6}$; $(775 \mathrm{mg}$, $60 \%$ ) colorless syrup; $[\alpha]_{\mathrm{D}}^{21}-12.5\left(c 1.0, \mathrm{CH}_{2} \mathrm{Cl}_{2}\right) ; R_{f}=0.16$ (solvent $\left.\mathrm{B}_{7}\right)$. ${ }^{1} \mathrm{H}$ NMR $(250 \mathrm{MHz}$, $\left.\mathrm{CDCl}_{3}\right) \delta 1.07\left[\mathrm{~s}, 9 \mathrm{H}, \mathrm{C}\left(\mathrm{CH}_{3}\right)_{3}\right] ; 1.35,1.54\left[2 \mathrm{x} \mathrm{s}, 6 \mathrm{H}, \mathrm{C}\left(\mathrm{CH}_{3}\right)_{2}\right] ; 1.71-1.94(\mathrm{~m}, 2 \mathrm{H}, \mathrm{H}-4) ; 2.66-$ 2.75 (m, 2H, H-3); 3.80 (m, 2H, H-5'); 3.82-3.86 (m, 1H, H-1'); 4.07 (q, $1 \mathrm{H},{ }^{3} J_{3^{\prime}, 4^{\prime}} 3.5,{ }^{3} J_{4^{\prime}, 5^{\prime}} 3.5$ Hz, H-4'); 4.28 (dd, $1 \mathrm{H},{ }^{3} J_{1^{\prime}, 2^{\prime}} 5.4$ Hz, ${ }^{3} J_{2^{\prime}, 3^{\prime}} 6.7 \mathrm{~Hz}, \mathrm{H}-2^{\prime}$ ); 4.74 (dd, 1H, H-3'); 7.28 (t, 1H, ${ }^{3} J_{2,3} 7.8$

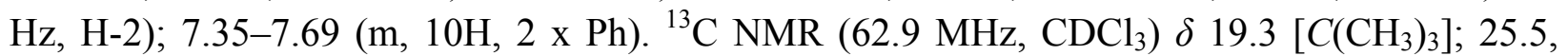
$27.5\left[\mathrm{C}\left(\mathrm{CH}_{3}\right)_{2}\right] ; 26.9\left[\mathrm{C}\left(\mathrm{CH}_{3}\right)_{3}\right] ; 29.7,31.4(\mathrm{C}-3, \mathrm{C}-4) ; 64.0\left(\mathrm{C}-5^{\prime}\right) ; 81.9\left(\mathrm{C}-3{ }^{\prime}\right) ; 83.5\left(\mathrm{C}-1^{\prime}\right) ; 84.4$ (C-4'); $84.5\left(\mathrm{C}-2^{\prime}\right) ; 110.4,112.0(2 \mathrm{CN}) ; 114.4\left[C\left(\mathrm{CH}_{3}\right)_{2}\right]$; 127.8, 127.8, 129.8, 129.9, 135.6 (2) (2 o-, $m-, p-\mathrm{Ph}) ; 133.1,133.2(2 i-\mathrm{Ph}) ; 169.0(\mathrm{C}-2)$. CI-MS: $m / z(\%)=517\left(3,[\mathrm{M}+\mathrm{H}]^{+}\right)$.

Anal. Calcd for $\mathrm{C}_{30} \mathrm{H}_{36} \mathrm{~N}_{2} \mathrm{O}_{4} \mathrm{Si}$ (516.70): C, 69.73; H, 7.02; N, 5.42. Found: C, 69.41; H, 7.03; N, 5.41 .

(E)-2-Cyano-5-(5-O-tert-butyldiphenylsilyl-2,3- $O$-isopropylidene-1-deoxy- $\beta$-D-ribofuranos1-yl)pent-2-enamide (3). Reaction time $16 \mathrm{~h}$; flash chromatography solvent $\mathrm{B}_{3} ;(682 \mathrm{mg}, 51 \%)$ colorless syrup; $[\alpha]_{\mathrm{D}}^{21}-12.7\left(c 1.0, \mathrm{CHCl}_{3}\right) ; R_{f}=0.17$ (solvent $\left.\mathrm{B}_{4}\right) .{ }^{1} \mathrm{H}$ NMR $\left(250 \mathrm{MHz}, \mathrm{CDCl}_{3}\right.$ ) $\delta \quad 1.06\left[\mathrm{~s}, 9 \mathrm{H}, \mathrm{C}\left(\mathrm{CH}_{3}\right)_{3}\right] ; 1.34,1.53\left[2 \mathrm{~s}, 6 \mathrm{H}, \mathrm{C}\left(\mathrm{CH}_{3}\right)_{2}\right] ; 1.78-1.88(\mathrm{~m}, 2 \mathrm{H}, \mathrm{H}-4) ; 2.63-2.72$ (m, $2 \mathrm{H}, \mathrm{H}-3) ; 3.78$ (m, 2H, H-5'); 3.82-3.89 (m, 1H, H-1'); 4.05 (q, $\left.1 \mathrm{H},{ }^{3} J_{3^{\prime}, 4^{\prime}} 3.6,{ }^{3} J_{4^{\prime}, 5^{\prime}} 3.6 \mathrm{~Hz}, \mathrm{H}-4^{\prime}\right)$; $4.30\left(\mathrm{dd}, 1 \mathrm{H},{ }^{3} J_{1^{\prime}, 2^{\prime}} 5.2 \mathrm{~Hz},{ }^{3} J_{2^{\prime}, 3^{\prime}} 6.7 \mathrm{~Hz}, \mathrm{H}-2^{\prime}\right) ; 4.72$ (dd, 1H, H-3'); 5.71, 6.09 (2 br s, 2H, NH$)$; 7.34-7.47, 7.61-7.73 (2m, 11H, $2 \mathrm{Ph}, \mathrm{H}-2) .{ }^{13} \mathrm{C}$ NMR (75.5 MHz, $\left.\mathrm{CDCl}_{3}\right) \delta 19.3\left[C\left(\mathrm{CH}_{3}\right)_{3}\right]$; 25.5, $27.5\left[\mathrm{C}\left(\mathrm{CH}_{3}\right)_{2}\right] ; 26.8\left[\mathrm{C}\left(\mathrm{CH}_{3}\right)_{3}\right] ; 28.5(\mathrm{C}-3) ; 31.8(\mathrm{C}-4) ; 64.0\left(\mathrm{C}-5^{\prime}\right) ; 81.9\left(\mathrm{C}-3^{\prime}\right) ; 83.4(\mathrm{C}-$ 1'); 84.2 (C-4'); 84.7 (C-2'); $110.1(\mathrm{C}-1) ; 114.3\left[C\left(\mathrm{CH}_{3}\right)_{2}\right]$; $114.9(\mathrm{CN}) ; 127.7,129.8,135.6(2$ o-, m-, $p$-Ph); 133.1, 133.2 (2 i-Ph); $160.8\left(\mathrm{CONH}_{2}\right) ; 161.3(\mathrm{C}-2)$. CI-MS: $m / z(\%)=535$ (3, $[\mathrm{M}+\mathrm{H}]^{+}$). Anal. Calcd for $\mathrm{C}_{30} \mathrm{H}_{38} \mathrm{~N}_{2} \mathrm{O}_{5} \mathrm{Si}$ (534.72): C, 67.39; H, 7.16; N, 5.24. Found: C, 67.64; H, 7.22; N, 5.27 .

(E)-2-Cyano-5-(5-O-tert-butyldiphenylsilyl-2,3- $O$-isopropylidene-1-deoxy- $\beta$-D-ribofuranos1-yl)- $\mathbf{N}$-(4-methoxyphenyl)pent-2-enamide (4). Reaction time $12 \mathrm{~h}$; flash chromatography solvent $\mathrm{B}_{5} ;(1.23 \mathrm{~g}, 77 \%)$ colorless syrup; $[\alpha]_{\mathrm{D}}^{21}-16.1\left(c 1.0, \mathrm{CHCl}_{3}\right) ; R_{f}=0.45$ (solvent $\left.\mathrm{B}_{4}\right)$.

${ }^{1} \mathrm{H}$ NMR $\left(250 \mathrm{MHz}, \mathrm{CDCl}_{3}\right) \delta 1.06\left[\mathrm{~s}, 9 \mathrm{H}, \mathrm{C}\left(\mathrm{CH}_{3}\right)_{3}\right] ; 1.35,1.54\left[2 \mathrm{~s}, 6 \mathrm{H}, \mathrm{C}\left(\mathrm{CH}_{3}\right)_{2}\right] ; 1.81-1.91$ (m, 2H, H-4); 2.67-2.76 (m, 2H, H-3); 3.79 (m, 2H, H-5'); 3.81 (s, 3H, $\left.\mathrm{OCH}_{3}\right) ; 3.84-3.91$ (m, $\left.1 \mathrm{H}, \mathrm{H}-1^{\prime}\right) ; 4.06$ (q, $\left.1 \mathrm{H},{ }^{3} J_{3^{\prime}, 4^{\prime}} 3.5 \mathrm{~Hz},{ }^{3} J_{4^{\prime}, 5^{\prime}} 3.5 \mathrm{~Hz}, \mathrm{H}-4^{\prime}\right) ; 4.32$ (dd, $1 \mathrm{H},{ }^{3} J_{1^{\prime}, 2^{\prime}} 5.3 \mathrm{~Hz},{ }^{3} J_{2^{\prime}, 3^{\prime}} 6.7 \mathrm{~Hz}$, H-2'); 4.74 (dd, 1H, H-3'); 6.87-6.93 (m, 2H, $\left.m-\mathrm{NHC}_{6} \mathrm{H}_{4}\right)$; 7.35-7.70 (m, 10H, 2 Ph); 7.44-7.49 $\left(\mathrm{m}, 2 \mathrm{H}, o-\mathrm{NHC}_{6} \mathrm{H}_{4}\right) ; 7.77\left(\mathrm{t}, 1 \mathrm{H},{ }^{3} J_{2,3} 7.9 \mathrm{~Hz}, \mathrm{H}-2\right) .{ }^{13} \mathrm{C} \mathrm{NMR}\left(75.5 \mathrm{MHz}, \mathrm{CDCl}_{3}\right) \delta 19.3$ $\left[\mathrm{C}\left(\mathrm{CH}_{3}\right)_{3}\right] ; 25.5,27.5\left[\mathrm{C}\left(\mathrm{CH}_{3}\right)_{2}\right] ; 26.8\left[\mathrm{C}\left(\mathrm{CH}_{3}\right)_{3}\right] ; 28.6(\mathrm{C}-3) ; 31.9(\mathrm{C}-4) ; 55.5\left(\mathrm{OCH}_{3}\right) ; 64.1(\mathrm{C}-$ $\left.5^{\prime}\right) ; 81.9$ (C-3'); $83.4\left(\mathrm{C}-1^{\prime}\right) ; 84.3$ (C-4'); 84.7 (C-2'); 111.1 (C-1); $114.3\left(m-\mathrm{NHC}_{6} \mathrm{H}_{4}\right) ; 114.3$ 
$\left[C\left(\mathrm{CH}_{3}\right)_{2}\right] ; 114.9(\mathrm{CN}) ; 122.4\left(o-\mathrm{NHC}_{6} \mathrm{H}_{4}\right) ; 129.7\left(i-\mathrm{NHC}_{6} \mathrm{H}_{4}\right) ; 127.7,129.8,135.5(2 o-, m-, p-$ $\mathrm{Ph}) ; 133.2,133.3$ (2 $i$-Ph); $157.2(\mathrm{CONH}) ; 160.7(\mathrm{C}-2)$. CI-MS: $m / z(\%)=641\left(8,[\mathrm{M}+\mathrm{H}]^{+}\right)$. Anal. Calcd for $\mathrm{C}_{37} \mathrm{H}_{44} \mathrm{~N}_{2} \mathrm{O}_{6} \mathrm{Si}$ (640.84): C, 69.35; H, 6.92; N, 4.37. Found: C, 69.14; H, 6.97; N, 4.24 .

(E)-2-Cyano-5-[3,5-O-(tetraisopropyldisiloxan-1,3-diyl)-1,2-dideoxy- $\beta$-D-ribofuranos-1-yl] pent-2-enamide (15). Starting from aldehyde 14 (1.04 g, $2.5 \mathrm{mmol})$ and 2-cyanoacetamide (479 $\mathrm{mg}, 5.7 \mathrm{mmol})$ Knoevenagel product 15 (628 $\mathrm{mg}$, 52\%) was obtained as colorless syrup according to the procedure described for compound 3; reaction time $24 \mathrm{~h}$; flash chromatography solvent $\mathrm{B}_{2} ;[\alpha]_{\mathrm{D}}^{22}-25.9\left(c 1.0, \mathrm{CH}_{2} \mathrm{Cl}_{2}\right) ; R_{f}=0.35$ (solvent $\left.\mathrm{B}_{2}\right) .{ }^{1} \mathrm{H}$ NMR $\left(250 \mathrm{MHz}, \mathrm{CDCl}_{3}\right) \delta$ 1.00-1.06 [m, 28H, $\left.4 \mathrm{CH}\left(\mathrm{CH}_{3}\right)_{2}\right] ; 1.65-1.77$ (m, 2H, H-4); 1.80 (dt, $1 \mathrm{H},{ }^{3} J_{1^{\prime}, 2^{\prime} \mathrm{a}} 7.8 \mathrm{~Hz},{ }^{3} J_{2^{\prime} \mathrm{a}, 3^{\prime}} 7.8$ $\left.\mathrm{Hz},{ }^{2} J_{2^{\prime} \mathrm{a}, 2^{\prime} \mathrm{b}} 12.5 \mathrm{~Hz}, \mathrm{H}-2^{\prime} \mathrm{a}\right) ; 2.05$ (ddd, $\left.1 \mathrm{H},{ }^{3} J_{2^{\prime} \mathrm{b}, 3^{\prime}} 4.5 \mathrm{~Hz},{ }^{3} J_{1^{\prime}, 2^{\prime} \mathrm{b}} 6.6 \mathrm{~Hz}, \mathrm{H}-2{ }^{\prime} \mathrm{b}\right) ; 2.63$ (m, 2H, H-3); 3.70 (m, 2H, H-5'a, H-4'); 4.00 (m, 1H, H-5'b); 4.07 (m, 1H, H-1'); 4.37 (dt, 1H, ${ }^{3} J_{3^{\prime}, 4^{\prime}} 7.8 \mathrm{~Hz}$, $\left.\mathrm{H}_{-3}\right)^{\prime} ; 5.80,6.18\left(2 \mathrm{x}\right.$ br s, $\left.2 \mathrm{H}, \mathrm{NH}_{2}\right) ; 7.69\left(\mathrm{t}, 1 \mathrm{H},{ }^{3} J_{2,3} 7.9 \mathrm{~Hz}, \mathrm{H}-2\right) .{ }^{13} \mathrm{C} \mathrm{NMR}(62.9 \mathrm{MHz}$, $\left.\mathrm{CDCl}_{3}\right) \delta 12.5,12.9,13.3,13.5\left[4 \mathrm{CH}\left(\mathrm{CH}_{3}\right)_{2}\right] ; 16.9,17.0,17.1,17.3$ (2), 17.4, 17.5 (2) [4 $\mathrm{CH}\left(\mathrm{CH}_{3}\right)_{2}$ ]; 28.6 (C-3); 33.5 (C-4); $40.2\left(\mathrm{C}-2^{\prime}\right) ; 63.7$ (C-5'); 73.4 (C-3'); 77.2 (C-1'); 86.0 (C-4'); $109.9(\mathrm{C}-1) ; 114.9(\mathrm{CN}) ; 161.0\left(\mathrm{CONH}_{2}\right) ; 161.8(\mathrm{C}-2)$. ESI-MS (-): $m / z=481\left(100,[\mathrm{M}-\mathrm{H}]^{-}\right)$.

Anal. Calcd. For $\mathrm{C}_{23} \mathrm{H}_{42} \mathrm{~N}_{2} \mathrm{O}_{5} \mathrm{Si}_{2}$ (482.76): C, 57.22; H, 8.77; N, 5.80. Found: C, 57.23; H, 8.74; N, 5.72 .

\section{Preparation of thiophenes 5, 7, 8, and 16 - General procedure}

Sulfur $(50 \mathrm{mg}, 1,6 \mathrm{mmol})$ and triethylamine $(0.22 \mathrm{~mL}, 1.6 \mu \mathrm{mol})$ were added to a stirred solution of compounds $\mathbf{2}, \mathbf{3}, \mathbf{4}$ or $\mathbf{1 5}(1.0 \mathrm{mmol})$ in dry $N, N$-dimethylformamide $(5.0 \mathrm{~mL})$. After stirring for $2 \mathrm{~h}$ at r.t. aq sat $\mathrm{NaCl}(75 \mathrm{~mL})$ was added, and the mixture was extracted with $\mathrm{CH}_{2} \mathrm{Cl}_{2}(3 \mathrm{x}$ $100 \mathrm{~mL})$. The combined organic phases were washed with water $(2 \times 75 \mathrm{~mL})$, dried and concentrated. The residue was purified by flash chromatography.

\section{2-Amino-5-[(5-O-tert-butyldiphenylsilyl-2,3- $O$-isopropylidene-1-deoxy- $\boldsymbol{\beta}$-D-ribofuranos-1-}

yl)methyl]thiophene-3-carbonitrile (5). Flash chromatography solvent $\mathrm{B}_{4}$; (439 $\mathrm{mg}, 80 \%$ ) yellow syrup; $[\alpha]_{\mathrm{D}}^{21}-20.1\left(c 1.0, \mathrm{CH}_{2} \mathrm{Cl}_{2}\right) ; R_{f}=0.42$ (solvent $\left.\mathrm{B}_{4}\right) .{ }^{1} \mathrm{H} \mathrm{NMR}\left(250 \mathrm{MHz}, \mathrm{CDCl}_{3}\right) \delta$ 1.07 [s, 9H, C( $\left.\left(\mathrm{CH}_{3}\right)_{3}\right] ; 1.34,1.53\left[2 \mathrm{~s}, 6 \mathrm{H}, \mathrm{C}\left(\mathrm{CH}_{3}\right)_{2}\right] ; 2.85\left(\mathrm{dd}, 1 \mathrm{H},{ }^{3} J_{1^{\prime \prime} \mathrm{a}, 1^{\prime}} 7.3 \mathrm{~Hz},{ }^{2} J_{1^{\prime \prime} \text { a, 1"b }} 15.3\right.$ Hz, H-1"a); 2.97 (dd, 1H, ${ }^{3} J_{1 " b, 1} 4.6$ Hz, H-1"b); 3.81 (m, 2H, H-5'); 4.02 (dt, 1H, H-1'); 4.08 ('q', $\left.1 \mathrm{H},{ }^{3} J_{3^{\prime}, 4^{\prime}} 3.6 \mathrm{~Hz},{ }^{3} J_{4^{\prime}, 5^{\prime}} 3.6 \mathrm{~Hz}, \mathrm{H}-4^{\prime}\right) ; 4.36$ (dd, $\left.1 \mathrm{H},{ }^{3} J_{2^{\prime}, 3^{\prime}} 6.7 \mathrm{~Hz},{ }^{3} J_{1^{\prime}, 2^{\prime}} 5.0 \mathrm{~Hz}, \mathrm{H}-2^{\prime}\right) ; 4.70$ (dd, $1 \mathrm{H}$, $\left.\mathrm{H}_{-3}\right)$ ); 6.45 (s, 1H, H-4); 7.34-7.71 (m, 10H, $\left.\left.2 \mathrm{Ph}\right) .{ }^{13} \mathrm{C} \mathrm{NMR} \mathrm{(75.5} \mathrm{MHz,} \mathrm{CDCl}_{3}\right) \delta 19.3$ $\left.\left[\mathrm{C}\left(\mathrm{CH}_{3}\right)_{3}\right] ; 25.5,27.5\left[\mathrm{C}\left(\mathrm{CH}_{3}\right)_{2}\right] ; 26.8\left[\mathrm{C}\left(\mathrm{CH}_{3}\right)_{3}\right] ; 33.7(\mathrm{C}-1 ") ; 64.1(\mathrm{C}-5)^{\prime}\right) ; 81.8\left(\mathrm{C}-3{ }^{\prime}\right) ; 83.7(\mathrm{C}-$ $\left.1^{\prime}\right) ; 84.1$ (C-4'); $84.6(\mathrm{C}-2 ') ; 87.3(\mathrm{C}-3) ; 114.3\left[C\left(\mathrm{CH}_{3}\right)_{2}\right] ; 115.6(\mathrm{CN}) ; 123.3(\mathrm{C}-4) ; 124.9(\mathrm{C}-5)$; 127.8, 129.8, 135.6 (2 o-, $m$-, $p$-Ph); 133.1, 133.2 (2 i-Ph); $161.7(\mathrm{C}-2)$. EI-MS: $m / z(\%)=548$ $\left(3,[\mathrm{M}]^{+}\right)$. Anal. Calcd for $\mathrm{C}_{30} \mathrm{H}_{36} \mathrm{~N}_{2} \mathrm{O}_{4} \mathrm{SSi}$ (548.77): C, 65.66; H, 6.61; N, 5.10; S, 5.84. Found: C, 65.45; H, 6.92; N, 4.94; S, 5.68.

2-Amino-5-[(5-O-tert-butyldiphenylsilyl-2,3- $\boldsymbol{O}$-isopropylidene-1-deoxy- $\boldsymbol{\beta}$-D-ribofuranos-1yl)methyl]thiophene-3-carboxamide (7). Flash chromatography solvent $\mathrm{B}_{1}$; (453 mg, 80\%) 
yellow crystals; m.p. $88-90{ }^{\circ} \mathrm{C}$ (ethyl acetate $-n$-heptane); $[\alpha]_{\mathrm{D}}^{21}-13.6\left(c 1.0, \mathrm{CHCl}_{3}\right) ; R_{f}=0.14$ (solvent $\mathrm{B}_{2}$ ). ${ }^{1} \mathrm{H}$ NMR $\left(250 \mathrm{MHz}, \mathrm{CDCl}_{3}\right) \delta 1.06\left[\mathrm{~s}, 9 \mathrm{H}, \mathrm{C}\left(\mathrm{CH}_{3}\right)_{3}\right] ; 1.34,1.53\left[2 \mathrm{~s}, 6 \mathrm{H}, \mathrm{C}\left(\mathrm{CH}_{3}\right)_{2}\right]$; 2.81-3.02 (m, 2H, H-1"); 3.81 (m, 2H, H-5'); 4.04-4.10 (m, 2H, H-1', H-4'); 4.38 (dd, $1 \mathrm{H},{ }^{3} \mathrm{~J}^{\prime}, 2^{\prime}$ $\left.5.0 \mathrm{~Hz},{ }^{3} J_{2^{\prime}, 3^{\prime}} 6.7 \mathrm{~Hz}, \mathrm{H}-2^{\prime}\right) ; 4.68$ (dd, $\left.1 \mathrm{H},{ }^{3} J_{3^{\prime}, 4^{\prime}} 3.7 \mathrm{~Hz}, \mathrm{H}-3^{\prime}\right) ; 5.55$ (br, $\left.\mathrm{NH}_{2}\right) ; 6.41$ (s, 1H, H-4); 7.33-7.72 (m, 10H, $2 \mathrm{Ph}$ ); one $\mathrm{NH}_{2}$ signal not detected. ${ }^{13} \mathrm{C}$ NMR $\left(75.5 \mathrm{MHz}, \mathrm{CDCl}_{3}\right) \delta 19.3$

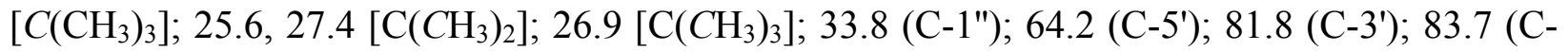
$\left.1^{\prime}\right) ; 84.3$ (C-4'); 84.6 (C-2'); 106.6 (C-3); $114.2\left[C\left(\mathrm{CH}_{3}\right)_{2}\right] ; 121.3$ (C-4); 121.7 (C-5); 127.8, 129.7, 129.7, $135.7(2 \quad o-, m-, p-\mathrm{Ph}) ; 133.1,133.3(2 i-\mathrm{Ph}) ; 161.7,167.7(\mathrm{C}-2, \mathrm{C}=\mathrm{O})$. $\mathrm{C}_{30} \mathrm{H}_{38} \mathrm{~N}_{2} \mathrm{O}_{5} \mathrm{SSi}$ (566.78); HRMS (EI): $\mathrm{m} / \mathrm{z}$ calculated for $\left[\mathrm{M}^{+}\right]=566.22679$, found 566.22652.

2-Amino-5-[(5-O-tert-butyldiphenylsilyl-2,3-O-isopropylidene-1-deoxy- $\beta$-D-ribofuranos-1yl)methyl]- $\boldsymbol{N}$-(4-methoxyphenyl)thiophene-3-carboxamide (8). Flash chromatography solvent $\mathrm{B}_{2} ;(511 \mathrm{mg}, 76 \%)$ yellow foam; $[\alpha]_{\mathrm{D}}^{23}-8.9\left(c 1.0, \mathrm{CH}_{2} \mathrm{Cl}_{2}\right) ; R_{f}=0.38$ (solvent $\left.\mathrm{B}_{2}\right)$. ${ }^{1} \mathrm{H}$ NMR $\left(250 \mathrm{MHz}, \mathrm{CDCl}_{3}\right) \delta 1.08\left[\mathrm{~s}, 9 \mathrm{H}, \mathrm{C}\left(\mathrm{CH}_{3}\right)_{3}\right] ; 1.36,1.55\left[2 \mathrm{~s}, 6 \mathrm{H}, \mathrm{C}\left(\mathrm{CH}_{3}\right)_{2}\right] ; 2.89\left(\mathrm{dd}, 1 \mathrm{H},{ }^{3} J_{1^{\prime \prime} \mathrm{a}, 1^{\prime}}\right.$

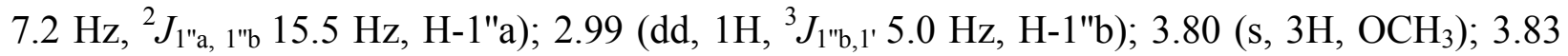
(m, 2H, H-5'); 4.07-4.16 (m, 2H, H-1', H-4'); 4.42 (dd, $\left.1 \mathrm{H},{ }^{3} J_{1^{\prime}, 2^{\prime}} 4.8 \mathrm{~Hz},{ }^{3} J_{2^{\prime}, 3^{\prime}} 6.7 \mathrm{~Hz}, \mathrm{H}-2^{\prime}\right) ; 4.72$ (dd, $\left.1 \mathrm{H},{ }^{3} J_{3^{\prime}, 4^{\prime}} 3.7, \mathrm{H}-3^{\prime}\right) ; 6.53$ (s, $\left.1 \mathrm{H}, \mathrm{H}-4\right) ; 6.86$ (m, $\left.2 \mathrm{H}, m-\mathrm{NHC}_{6} \mathrm{H}_{4}\right) ; 7.18$ (br s, $\left.\mathrm{NH}_{2}\right) ; 7.35$ (m, $\left.2 \mathrm{H}, o-\mathrm{NHC}_{6} \mathrm{H}_{4}\right) ; 7.35-7.70(\mathrm{~m}, 10 \mathrm{H}, 2 \mathrm{Ph}) .{ }^{13} \mathrm{C} \mathrm{NMR}\left(62.9 \mathrm{MHz}, \mathrm{CDCl}_{3}\right) \delta 19.3\left[\mathrm{C}_{\left.\left(\mathrm{CH}_{3}\right)_{3}\right]}\right]$ 25.6, $27.5\left[\mathrm{C}\left(\mathrm{CH}_{3}\right)_{2}\right] ; 26.9\left[\mathrm{C}\left(\mathrm{CH}_{3}\right)_{3}\right] ; 33.9(\mathrm{C}-1 ") ; 55.5\left(\mathrm{OCH}_{3}\right) ; 64.2\left(\mathrm{C}-5^{\prime}\right) ; 81.8\left(\mathrm{C}-3^{\prime}\right) ; 83.8$

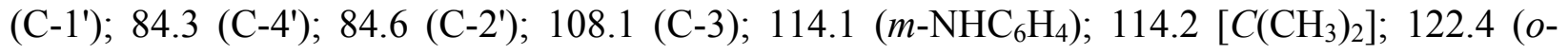
$\left.\mathrm{NHC}_{6} \mathrm{H}_{4}\right) ; 127.7,127.8,129.7,129.8,135.6$ (2) $(2 \quad o-, m-, p-\mathrm{Ph}) ; 130.8\left(i-\mathrm{NHC}_{6} \mathrm{H}_{4}\right) ; 133.2$, $133.3(2 i-\mathrm{Ph}) ; 156.3(\mathrm{C}=\mathrm{O}) ; 160.9,163.9\left(\mathrm{C}-2, p-\mathrm{NHC}_{6} \mathrm{H}_{4}\right) . \mathrm{C}_{37} \mathrm{H}_{44} \mathrm{~N}_{2} \mathrm{O}_{6} \mathrm{SSi}$ (672.91); HRMS (EI): $\mathrm{m} / \mathrm{z}$ calculated for $\left[\mathrm{M}^{+}\right]=672.26814$, found 672.26830 .

2-Amino-5-[[3,5-O-(tetraisopropyldisiloxan-1,3-diyl)-1,2-dideoxy- $\beta$-D-ribofuranos-1yl]methyl]thiophene-3-carboxamide (16). Flash chromatography solvent $\mathrm{B}_{2}$; (398 $\left.\mathrm{mg}, 73 \%\right)$ yellow syrup; $[\alpha]_{\mathrm{D}}^{24}-25.8\left(c 1.0, \mathrm{CH}_{2} \mathrm{Cl}_{2}\right) ; R_{f}=0.20$ (solvent $\left.\mathrm{B}_{2}\right) .{ }^{1} \mathrm{H} \mathrm{NMR}\left(250 \mathrm{MHz}, \mathrm{CDCl}_{3}\right) \delta$ $1.00-1.08$ [m, 28H, $\left.4 \mathrm{CH}\left(\mathrm{CH}_{3}\right)_{2}\right] ; 1.87\left(\mathrm{dt}, 1 \mathrm{H},{ }^{3} J_{1^{\prime}, 2^{\prime} \mathrm{a}} 7.9 \mathrm{~Hz},{ }^{3} J_{2^{\prime} \mathrm{a}, 3^{\prime}} 7.9 \mathrm{~Hz},{ }^{2} J_{2^{\prime} \mathrm{a}, 2^{\prime} \mathrm{b}} 12.9 \mathrm{~Hz}, \mathrm{H}-\right.$ 2'a); 2.02 (ddd, $1 \mathrm{H},{ }^{3} J_{2 ' b, 3^{\prime}} 4.4 \mathrm{~Hz},{ }^{3} J_{1^{\prime}, 2^{\prime} \mathrm{b}} 6.6 \mathrm{~Hz}, \mathrm{H}-2^{\prime} \mathrm{b}$ ); 2.80 (m, 2H, H-1"); 3.70-3.79 (m, 2H, H-5'a, H-4'); 4.04 (m, 1H, H-5'b); 4.23 (m, 1H, H-1'); 4.33 (dt, 1H, $\left.{ }^{3} J_{3^{\prime}, 4^{\prime}} 7.9 \mathrm{~Hz}, \mathrm{H}-3^{\prime}\right) ; 5.37$ (br s, $\left.2 \mathrm{H}, \mathrm{NH}_{2}\right) ; 6.06$ (br s, $\left.2 \mathrm{H}, \mathrm{NH}_{2}\right) ; 6.42(\mathrm{~s}, 1 \mathrm{H}, \mathrm{H}-4) .{ }^{13} \mathrm{C} \mathrm{NMR}\left(62.9 \mathrm{MHz}, \mathrm{CDCl}_{3}\right) \delta 12.5,13.0$, 13.3, $13.4\left[4 \mathrm{CH}\left(\mathrm{CH}_{3}\right)_{2}\right] ; 16.9,17.0,17.1,17.2,17.3,17.4,17.5,17.6\left[4 \mathrm{CH}\left(\mathrm{CH}_{3}\right)_{2}\right] ; 35.4(\mathrm{C}-1 ")$; 39.5 (C-2'); 64.0 (C-5'); 73.8 (C-3'); 77.3 (C-1'); 86.2 (C-4'); 107.1 (C-3); 121.4 (C-4); 121.5 (C5); 161.4, $167.6(\mathrm{C}-2, \mathrm{C}=\mathrm{O})$. ESI-MS $(-): m / z=513\left(100,[\mathrm{M}-\mathrm{H}]^{-}\right)$. Anal. Calcd for $\mathrm{C}_{23} \mathrm{H}_{42} \mathrm{~N}_{2} \mathrm{O}_{5} \mathrm{SSi}_{2}$ (514.83): C, 53.66; H, 8.22; N, 5.44; $\mathrm{S}, 6.23$. Found: $\mathrm{C}, 53.38 ; \mathrm{H}, 8.41 ; \mathrm{N}$ 5.21; $\mathrm{S} ; 5.94$.

\section{4-Amino-6-[(5-O-tert-butyldiphenylsilyl-2,3- $O$-isopropylidene-1-deoxy- $\beta$-D-ribofuranos-1- yl)methyl]thieno[2,3-d]pyrimidine (6)}

A mixture of compound $\mathbf{5}(165 \mathrm{mg}, 0.3 \mathrm{mmol})$ and triethylorthoformiate $(3.0 \mathrm{~mL}, 18 \mathrm{mmol})$ was heated under reflux for $2 \mathrm{~h}$. The reaction mixture was concentrated and the obtained syrup was dissolved in a solution of ethanol-ammonia $(1: 1,6.0 \mathrm{~mL})$. After heating under reflux for $2 \mathrm{~h}$ the 
mixture was allowed to attain r.t. and then concentrated. Purification by flash chromatography solvent $\mathrm{B}_{2}$ afforded compound $6(128 \mathrm{mg}, 74 \%)$ as a colorless foam, $[\alpha]_{\mathrm{D}}^{21}-15.5\left(c 1.0, \mathrm{CHCl}_{3}\right)$; $\mathrm{R}_{f}=0.18$ (solvent $\mathrm{B}_{2}$ ).

${ }^{1} \mathrm{H}$ NMR $\left(250 \mathrm{MHz}, \mathrm{CDCl}_{3}\right) \delta 1.07\left[\mathrm{~s}, 9 \mathrm{H}, \mathrm{C}\left(\mathrm{CH}_{3}\right)_{3}\right] ; 1.35,1.54\left[2 \mathrm{~s}, 6 \mathrm{H}, \mathrm{C}\left(\mathrm{CH}_{3}\right)_{2}\right] ; 3.12$ (dd, $1 \mathrm{H},{ }^{3} J_{1 ' 1 " \mathrm{a}} 7.9 \mathrm{~Hz},{ }^{2} J_{1 " \mathrm{a}, 1 " \mathrm{~b}} 15.3 \mathrm{~Hz}, \mathrm{H}-1$ "a); 3.23 (dd, 1H, ${ }^{3} J_{1 ' 1 " \mathrm{l}} 4.8 \mathrm{~Hz}, \mathrm{H}-1$ "b); 3.82 (m, 2H, H$\left.5^{\prime}\right) ; 4.13$ (q, $\left.1 \mathrm{H},{ }^{3} J_{3^{\prime}, 4^{\prime}} 3.6 \mathrm{~Hz},{ }^{3} J_{4^{\prime}, 5^{\prime}} 3.6 \mathrm{~Hz}, \mathrm{H}-4^{\prime}\right) ; 4.22$ (dt, $\left.1 \mathrm{H},{ }^{3} J_{1^{\prime}, 2^{\prime}} 4.8 \mathrm{~Hz}, \mathrm{H}-1^{\prime}\right) ; 4.44$ (dd, $1 \mathrm{H}$, $\left.{ }^{3} J_{2^{\prime}, 3^{\prime}} 6.7 \mathrm{~Hz}, \mathrm{H}-2^{\prime}\right) ; 4.74$ (dd, 1H, H-3'); 5.50 (br s, NH ); 6.88 (s, 1H, H-5); 7.33-7.72 (m, 10H,

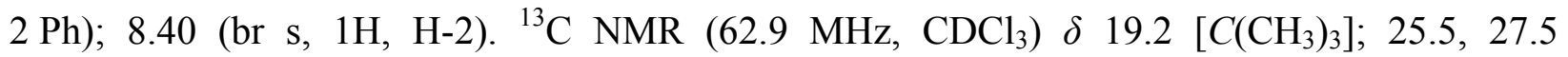
$\left[\mathrm{C}\left(\mathrm{CH}_{3}\right)_{2}\right] ; 26.8\left[\mathrm{C}\left(\mathrm{CH}_{3}\right)_{3}\right] ; 34.9\left(\mathrm{C}-1^{\prime \prime}\right) ; 64.2(\mathrm{C}-5 ') ; 81.9\left(\mathrm{C}-3^{\prime}\right) ; 84.1\left(\mathrm{C}-1^{\prime}\right) ; 84.2\left(\mathrm{C}-4^{\prime}\right) ; 84.7$ $\left(\mathrm{C}-2^{\prime}\right) ; 114.3\left[\mathrm{C}\left(\mathrm{CH}_{3}\right)_{2}\right] ; 115.7(\mathrm{C}-5) ; 115.7$ (C-4a); 127.7, 127.8, 129.8, 129.9, 135.6 (2) (2 o-, $m-, p-\mathrm{Ph}) ; 133.1,133.2$ (2 i-Ph); 138.8 (C-6); 152.7 (C-2); 156.7 (C-7a); 167.4 (C-4).

EI-MS: $m / z(\%)=576\left(3,[\mathrm{M}+\mathrm{H}]^{+}\right)$. Anal. Calcd for $\mathrm{C}_{31} \mathrm{H}_{37} \mathrm{~N}_{3} \mathrm{O}_{4} \mathrm{SSi}$ (575.79): C, 64.66; H, 6.48; N, 7.30; S, 5.57. Found: C, 64.66; H, 6.91; N, 7.12; S, 5.71.

\section{Preparation of thienopyrimidinones $(9,10$, and 17). General procedure}

Triethylorthoformiate $(0.25 \mathrm{~mL}, 1.5 \mathrm{mmol})$ was added to a solution of compound 7 (283 $\mathrm{mg}, 0.5$ $\mathrm{mmol}), 8(337 \mathrm{mg}, 0.5 \mathrm{mmol})$, or $16(257 \mathrm{mg}, 0.5 \mathrm{mmol})$ in dry DMF $(5.25 \mathrm{~mL})$ and the reaction mixture was heated under reflux for $7-10 \mathrm{~h}$ (monitored by TLC). After cooling to r.t. the mixture was concentrated and the residue was purified by flash chromatography.

6-[(5-O-tert-Butyldiphenylsilyl-2,3- $O$-isopropylidene-1-deoxy- $\beta$-D-ribofuranos-1-yl)methyl] thieno[2,3-d]pyrimidin-4(3H)-one (9). Reaction time $7 \mathrm{~h}$; flash chromatography solvent $\mathrm{B}_{1}$; (205 mg, 72\%) light yellow solid; m.p. $71-73{ }^{\circ} \mathrm{C}$; $[\alpha]_{\mathrm{D}}^{22}-22.5\left(c 0.9, \mathrm{CHCl}_{3}\right) ; R_{f}=0.13$ (solvent $\left.\mathrm{B}_{2}\right) .{ }^{1} \mathrm{H} \mathrm{NMR}\left(250 \mathrm{MHz}, \mathrm{CDCl}_{3}\right) \delta 1.07\left[\mathrm{~s}, 9 \mathrm{H}, \mathrm{C}\left(\mathrm{CH}_{3}\right)_{3}\right] ; 1.34,1.53\left[2 \mathrm{~s}, 6 \mathrm{H}, \mathrm{C}\left(\mathrm{CH}_{3}\right)_{2}\right] ; 3.09-$ 3.29 (m, 2H, H-1"); 3.82 (m, 2H, H-5'); 4.11-4.21 (m, 2H, H-4', H-1'); 4.44 (dd, 1H, ${ }^{3} J_{1^{\prime}, 2^{\prime}} 5.0 \mathrm{~Hz}$, $\left.{ }^{3} J_{2^{\prime}, 3^{\prime}} 6.6 \mathrm{~Hz}, \mathrm{H}-2^{\prime}\right) ; 4.74$ (dd, $\left.1 \mathrm{H},{ }^{3} J_{3^{\prime}, 4^{\prime}} 3.5 \mathrm{~Hz}, \mathrm{H}-3^{\prime}\right) ; 7.29$ (s, 1H, H-5); 7.33-7.72 (m, 10H, 2

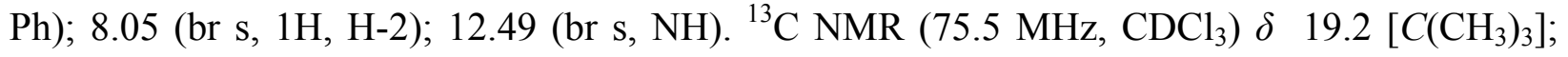
25.6, $27.5\left[\mathrm{C}\left(\mathrm{CH}_{3}\right)_{2}\right] ; 26.9\left[\mathrm{C}\left(\mathrm{CH}_{3}\right)_{3}\right] ; 34.8\left(\mathrm{C}-1^{\prime \prime}\right) ; 64.1\left(\mathrm{C}-5^{\prime}\right) ; 82.0\left(\mathrm{C}-3{ }^{\prime}\right) ; 84.0\left(\mathrm{C}-1^{\prime}\right)$; $84.2(\mathrm{C}-$ $\left.4^{\prime}\right)$; $84.7\left(\mathrm{C}-2^{\prime}\right) ; 114.3\left[\mathrm{C}\left(\mathrm{CH}_{3}\right)_{2}\right] ; 119.6(\mathrm{C}-5) ; 124.7(\mathrm{C}-4 \mathrm{a}) ; 127.7,127.8,129.7,129.8,135.6$ (2) $(2 o-, m-, p-\mathrm{Ph}) ; 133.0,133.1$ (2 i-Ph); 139.4 (C-6); 143.1 (C-2); 159.5 (C-7a); 165.0 (C-4).

CI-MS: $m / z(\%)=577\left(6,[\mathrm{M}+\mathrm{H}]^{+}\right)$. Anal Calcd for $\mathrm{C}_{31} \mathrm{H}_{36} \mathrm{~N}_{2} \mathrm{O}_{5} \mathrm{SSi}$ (576.21): C, 64.55; H, 6.29; N, 4.86; S, 5.56. Found: C, 64.28; H, 6.49; N, 4.64; S, 5.35.

\section{6-(5-O-tert-Butyldiphenylsilyl-2,3-O-isopropylidene-1-deoxy- $\beta$-D-ribofuranos-1-yl)methyl)-}

3-(4-methoxyphenyl)thieno[2,3-d]pyrimidin-4(3H)-one (10). Reaction time $7 \mathrm{~h}$; flash chromatography solvent $\mathrm{B}_{4} ;(236 \mathrm{mg}, 69 \%)$ yellow syrup; $[\alpha]_{\mathrm{D}}^{24}-14.4\left(c 1.0, \mathrm{CH}_{2} \mathrm{Cl}_{2}\right) ; R_{f}=0.42$

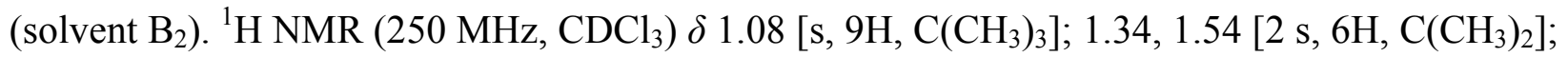
$3.12\left(\mathrm{dd}, 1 \mathrm{H},{ }^{3} J_{1^{\prime}, 1 " \mathrm{a}} 7.5 \mathrm{~Hz},{ }^{2} J_{1^{\prime \prime}, 11^{\prime b}} 15.3 \mathrm{~Hz}, \mathrm{H}-1 " \mathrm{a}\right) ; 3.22$ (dd, $\left.1 \mathrm{H},{ }^{3} J_{1^{\prime}, 1^{\prime \prime}} 5.0 \mathrm{~Hz}, \mathrm{H}-1 " \mathrm{~b}\right) ; 3.82$ (m, $\left.2 \mathrm{H}, \mathrm{H}-5^{\prime}\right) ; 3.87$ (s, 3H, $\left.\mathrm{OCH}_{3}\right) ; 4.12$ (q, $\left.1 \mathrm{H},{ }^{3} J_{3^{\prime}, 4^{\prime}} 3.5 \mathrm{~Hz},{ }^{3} J_{4^{\prime}, 5^{\prime}} 3.5 \mathrm{~Hz}, \mathrm{H}-4^{\prime}\right) ; 4.17$ (dt, $1 \mathrm{H},{ }^{3} J_{1^{\prime}, 2^{\prime}}$ $\left.5.0 \mathrm{~Hz}, \mathrm{H}-1^{\prime}\right) ; 4.44$ (dd, 1H, $\left.{ }^{3} J_{2^{\prime}, 3^{\prime}} 6.6 \mathrm{~Hz}, \mathrm{H}-2^{\prime}\right) ; 4.74$ (dd, 1H, H-3'); 7.03 (m, 2H, m-NHC $\mathrm{H}_{4}$ ); $7.30\left(\mathrm{~m}, 2 \mathrm{H}, o-\mathrm{NHC}_{6} \mathrm{H}_{4}\right) ; 7.31$ (s, 1H, H-5); 7.37-7.72 (m, 10H, $\left.2 \mathrm{Ph}\right) ; 8.02$ (s, 1H, H-2). 
${ }^{13} \mathrm{C} \mathrm{NMR}\left(62.9 \mathrm{MHz}, \mathrm{CDCl}_{3}\right) \delta 19.3\left[\mathrm{C}\left(\mathrm{CH}_{3}\right)_{3}\right] ; 25.6,27.5\left[\mathrm{C}\left(\mathrm{CH}_{3}\right)_{2}\right] ; 26.9\left[\mathrm{C}\left(\mathrm{CH}_{3}\right)_{3}\right] ; 34.9(\mathrm{C}-$ $\left.1^{\prime \prime}\right) ; 55.6\left(\mathrm{OCH}_{3}\right) ; 64.2\left(\mathrm{C}-5^{\prime}\right) ; 82.0\left(\mathrm{C}-3^{\prime}\right) ; 84.0\left(\mathrm{C}-1^{\prime}\right) ; 84.4\left(\mathrm{C}-4^{\prime}\right) ; 84.7\left(\mathrm{C}-2^{\prime}\right) ; 114.2\left[\mathrm{C}^{\prime}\left(\mathrm{CH}_{3}\right)_{2}\right]$; $114.8\left(m-\mathrm{NHC}_{6} \mathrm{H}_{4}\right) ; 120.7(\mathrm{C}-5) ; 124.8(\mathrm{C}-4 \mathrm{a}) ; 127.8,128.2,135.6(2 o-, m-, p-\mathrm{Ph}) ; 129.8(o-$ $\left.\mathrm{NHC}_{6} \mathrm{H}_{4}\right) ; 129.9\left(i-\mathrm{NHC}_{6} \mathrm{H}_{4}\right) ; 133.0,133.1$ (2 $i$-Ph); 139.3 (C-6); 145.9 (C-2); 157.2 (C-7a); $160.0\left(p-\mathrm{NHC}_{6} \mathrm{H}_{4}\right) ; 162.9(\mathrm{C}-4)$. CI-MS: $m / z(\%)=683\left(100,[\mathrm{M}+\mathrm{H}]^{+}\right) . \mathrm{C}_{38} \mathrm{H}_{42} \mathrm{~N}_{2} \mathrm{O}_{6} \mathrm{SSi}$ (682.90); HRMS (EI): $\mathrm{m} / \mathrm{z}$ calculated for $\left[\mathrm{M}^{+}\right]=682.25328$, found 682.25341 .

6-[[3,5-O-(tetraisopropyldisiloxan-1,3-diyl)-1,2-dideoxy- $\beta$-D-ribofuranos-1-yl]methyl]

thieno[2,3- $d$ ]pyrimidin-4(3H)-one (17). Reaction time $10 \mathrm{~h}$; flash chromatography solvent $\mathrm{B}_{2}$; (181 mg, 69\%) colorless crystals; m.p. $150-152{ }^{\circ} \mathrm{C}$ (ethyl acetate $-n$-heptane); $[\alpha]_{\mathrm{D}}^{24}-17.0(c$ $1.0, \mathrm{CH}_{2} \mathrm{Cl}_{2}$ ); $R_{f}=0.20$ (solvent $\mathrm{B}_{2}$ ). ${ }^{1} \mathrm{H}$ NMR (250 $\left.\mathrm{MHz}, \mathrm{CDCl}_{3}\right) \delta 0.98-1.05[\mathrm{~m}, 28 \mathrm{H}, 4$ $\left.\mathrm{CH}\left(\mathrm{CH}_{3}\right)_{2}\right] ; 1.88\left(\mathrm{dt}, 1 \mathrm{H},{ }^{3} J_{1^{\prime}, 2^{\prime} \mathrm{a}} 7.9 \mathrm{~Hz},{ }^{3} J_{2^{\prime} \mathrm{a}, 3^{\prime}} 7.9 \mathrm{~Hz},{ }^{2} J_{2^{\prime} \mathrm{a}, 2^{\prime} \mathrm{b}} 13.0 \mathrm{~Hz}, \mathrm{H}-2{ }^{\prime} \mathrm{a}\right) ; 2.05$ (ddd, $1 \mathrm{H},{ }^{3} J_{2^{\prime} \mathrm{b}, 3^{\prime}}$ $\left.4.5 \mathrm{~Hz},{ }^{3} J_{1^{\prime}, 2^{\prime} \mathrm{b}} 6.6 \mathrm{~Hz}, \mathrm{H}-2^{\prime} \mathrm{b}\right) ; 3.07$ (m, 2H, H-1"); 3.69-3.81 (m, 2H, H-5'a, H-4'); 4.05 (m, 1H, H-5'b); 4.34 (m, 2H, H-1', H-3'); 7.22 (s, 1H, H-5); 8.03 (s, 1H, H-2); 12.77 (br s, 1H, NH).

${ }^{13} \mathrm{C} \mathrm{NMR}\left(62.9 \mathrm{MHz}, \mathrm{CDCl}_{3}\right) \delta 12.5,12.9,13.3,13.4\left[4 \mathrm{CH}\left(\mathrm{CH}_{3}\right)_{2}\right] ; 16.9,17.0,17.1,17.2,17.3$, 17.4, 17.5, 17.6 [4 CH( $\left.\left(\mathrm{CH}_{3}\right)_{2}\right] ; 36.3\left(\mathrm{C}-1^{\prime \prime}\right) ; 39.6$ (C-2'); 63.9 (C-5'); 73.7 (C-3'); 76.8 (C-1'); 86.4 (C-4'); 119.6 (C-5); 124.6 (C-4a); 139.3 (C-6); 143.1 (C-2); 159.9 (C-7a); 165.3 (C-4).

Anal. Calcd for $\mathrm{C}_{24} \mathrm{H}_{40} \mathrm{~N}_{2} \mathrm{O}_{5} \mathrm{SSi}_{2}$ (524.82): C, 54.92; H, 7.68; N, 5.44; S, 6.11. Found: C, 54.97, $\mathrm{H}, 7.82 ; \mathrm{N}, 5.26$, S; 5.83 .

\section{Deprotection of compounds (6) and (9)}

90\% Trifluoroacetic acid $(25 \mathrm{~mL})$ was added to a solution of compound 6 (576 mg, $1.0 \mathrm{mmol})$ or compound 9 (576 mg, $1.0 \mathrm{mmol})$ in $\mathrm{CH}_{2} \mathrm{Cl}_{2}(10 \mathrm{~mL})$. After stirring at r.t. (monitored by TLC), the reaction mixture was concentrated. Traces of acid were removed by evaporation with repeated addition of toluene. The residue was then subjected to flash chromatography.

\section{4-Amino-6-[(1-deoxy- $\beta$-D-ribofuranos-1-yl)methyl]thieno[2,3- $\boldsymbol{d}]$ pyrimidine (11)}

Flash chromatography solvent $\mathrm{C}_{2} ;(268 \mathrm{mg}, 90 \%)$ colorless foam; $[\alpha]_{\mathrm{D}}^{21}-7.8(c 1.0, \mathrm{MeOH}) ; R_{f}=$ 0.14 (solvent $\left.\mathrm{C}_{2}\right)$. ${ }^{1} \mathrm{H}$ NMR $\left(300 \mathrm{MHz}, \mathrm{DMSO}-d_{6}\right) \delta 2.92\left(\mathrm{dd}, 1 \mathrm{H},{ }^{3} J_{1 " \mathrm{a}, 1}, 7.8 \mathrm{~Hz},{ }^{2} J_{1 \mathrm{an}, 1 " \mathrm{~b}} 15.2 \mathrm{~Hz}\right.$, H-1"a); 3.13 (dd, $\left.1 \mathrm{H},{ }^{3} J_{1^{\prime \prime} \mathrm{b}, 1^{\prime}} 3.5 \mathrm{~Hz}, \mathrm{H}-1^{\prime \prime} \mathrm{b}\right) ; 3.39$ (m, 2H, H-5'); 3.60 (q, 1H, ${ }^{3} J_{1^{\prime}, 2^{\prime}} 6.2 \mathrm{~Hz},{ }^{3} J_{2^{\prime}, 3^{\prime}}$ $\left.6.2 \mathrm{~Hz},{ }^{3} J_{2^{\prime}, \mathrm{OH}} 6.2 \mathrm{~Hz}, \mathrm{H}-2^{\prime}\right) ; 3.66$ (q, $\left.1 \mathrm{H},{ }^{3} J_{3^{\prime}, 4^{\prime}} 4.5 \mathrm{~Hz},{ }^{3} J_{4^{\prime}, 5^{\prime}} 4.5 \mathrm{~Hz}, \mathrm{H}-4^{\prime}\right) ; 3.73$ (m, 1H, H-3'); 3.83 (m, 1H, H-1'); 4.65 (t, $\left.1 \mathrm{H},{ }^{3} J_{5^{\prime}, \mathrm{OH}} 5.6 \mathrm{~Hz}, \mathrm{OH}-5^{\prime}\right) ; 4.81$ (d, 1H, $\left.{ }^{3} J_{3^{\prime}, \mathrm{OH}} 5.1 \mathrm{~Hz}, \mathrm{OH}-3^{\prime}\right) ; 4.88$ (d, 1H, OH-2'); 7.29 (s, 1H, H-5); 7.34 (br s, NH $) ; 8.18$ (s, 1H, H-2). ${ }^{13} \mathrm{C}$ NMR (75.5 MHz, DMSO-D $\left._{6}\right) \delta 34.3$ (C-1"); $62.0\left(\mathrm{C}^{\prime} 5^{\prime}\right) ; 71.1\left(\mathrm{C}-3^{\prime}\right) ; 73.8$ (C-2'); 81.6 (C-1'); $85.0\left(\mathrm{C}-4{ }^{\prime}\right) ; 115.7$ (C4a); 117.6 (C-5); 136.9 (C-6); 153.3 (C-2); 157.7 (C-7a); 165.9 (C-4). EI-MS: $m / z(\%)=297$ (15, $\left.[\mathrm{M}]^{+}\right)$. Anal. Calcd for $\mathrm{C}_{12} \mathrm{H}_{15} \mathrm{~N}_{3} \mathrm{O}_{4} \mathrm{~S}$ (297.33): C, 48.47; H, 5.08; N, 14.13; S, 10.78. Found: C, 48.30; H, 4.94; N, 14.23; S, 10.49 .

6-[(1-Deoxy- $\beta$-D-ribofuranos-1-yl)methyl]thieno[2,3- $d]$ pyrimidin-4(3H)-one $\quad(13) . \quad$ Flash chromatography solvent $\mathrm{C}_{2} ;(268 \mathrm{mg}, 90 \%)$ colorless foam; $[\alpha]_{\mathrm{D}}^{21}-7.5(c 1.1, \mathrm{MeOH}) ; R_{f}=0.20$ (solvent $\mathrm{C}_{2}$ ). ${ }^{1} \mathrm{H}$ NMR (300 MHz, DMSO-d $) \delta 2.94\left(\mathrm{dd}, 1 \mathrm{H},{ }^{3} J_{1^{\prime \prime} \mathrm{a}, 1^{\prime}} 7.6 \mathrm{~Hz},{ }^{2} J_{1^{\prime \prime}, 1^{\prime \prime b}} 15.3 \mathrm{~Hz}, \mathrm{H}-\right.$ 
$1 " \mathrm{a}) ; 3.12$ (dd, $\left.1 \mathrm{H},{ }^{3} J_{1^{\prime \prime} \mathrm{b}, 1^{\prime}} 3.8 \mathrm{~Hz}, \mathrm{H}-1^{\prime \prime} \mathrm{b}\right) ; 3.38-3.42$ (m, 2H, H-5'); 3.59 (q, $1 \mathrm{H},{ }^{3} J_{1^{\prime}, 2^{\prime}} 6.1 \mathrm{~Hz},{ }^{3} J_{2^{\prime}, 3^{\prime}}$ $\left.6.1 \mathrm{~Hz},{ }^{3} J_{2^{\prime}, \mathrm{OH}} 6.1 \mathrm{~Hz}, \mathrm{H}-2^{\prime}\right) ; 3.66$ (q, $\left.1 \mathrm{H},{ }^{3} J_{4^{\prime}, 3^{\prime}} 4.4 \mathrm{~Hz},{ }^{3} J_{4^{\prime}, 5^{\prime}} 4.4 \mathrm{~Hz}, \mathrm{H}-4^{\prime}\right) ; 3.71$ (m, 1H, H-3'); 3.81 (ddd, 1H, H-1'); 4.66 (t, 1H, $\left.{ }^{3} J_{5^{\prime}, \mathrm{OH}} 5.6 \mathrm{~Hz}, \mathrm{OH}-5^{\prime}\right) ; 4.79$ (d, $\left.1 \mathrm{H},{ }^{3} J_{3^{\prime}, \mathrm{OH}} 5.1 \mathrm{~Hz}, \mathrm{OH}-3{ }^{\prime}\right) ; 4.87$ $\left(\mathrm{d}, 1 \mathrm{H}, \mathrm{OH}-2^{\prime}\right) ; 7.15$ (s, 1H, H-5); 8.04 (s, 1H, H-2); 12.40 (br s, NH). ${ }^{13} \mathrm{C} \mathrm{NMR}(75.5 \mathrm{MHz}$, DMSO-D 6 ) $\delta 33.8$ (C-1"); 62.1 (C-5'); 71.2 (C-3'); 73.7 (C-2'); 81.6 (C-1'); 84.9 (C-4'); 119.7 (C5); 124.5 (C-4a); 138.5 (C-6); 145.0 (C-2); 157.1 (C-7a); 163.5 (C-4). CI-MS: m/z (\%) = 299 (6, $\left.[\mathrm{M}+\mathrm{H}]^{+}\right)$. Anal. Calcd for $\mathrm{C}_{12} \mathrm{H}_{14} \mathrm{~N}_{2} \mathrm{O}_{5} \mathrm{~S}$ (298.31): C, 48.31; H, 4.73; N, 9.39; S, 10.75. Found: C, 48.52; H, 4.64; N, 9.12; S, 10.71 .

\section{2-Amino-5-[(1-desoxy- $\beta$-D-ribofuranos-1-yl)methyl]thiophene-3-carbonitrile (12)}

A solution of tetrabutylammonium fluoride (TBAF) in 1,4-dioxane (1.0 M, 0.8 mL) was added dropwise to a solution of compound $5(283 \mathrm{mg}, 0.5 \mathrm{mmol})$ in 1,4-dioxane $(7 \mathrm{~mL})$. The reaction mixture was stirred at r.t. for $5 \mathrm{~h}$, and then concentrated. The residue was dissolved in $\mathrm{CH}_{2} \mathrm{Cl}_{2}$ (4 $\mathrm{mL}$ ) and a solution $90 \%$ trifluoroacetic acid $(6 \mathrm{~mL})$ was added. After stirring at r.t. (monitored by TLC), the reaction mixture was concentrated. Traces of acid were removed by evaporation with repeated addition of toluene. Purification by flash chromatography (solvent $\mathrm{C}_{4}$ ) afforded compound 12 (118 mg; 87\%) as yellow foam; $[\alpha]_{\mathrm{D}}^{25}-32.9(c 0.5, \mathrm{MeOH}) ; R_{f}=0.31$ (solvent D). ${ }^{1} \mathrm{H}$ NMR (300 MHz, CD $\left.{ }_{3} \mathrm{OD}\right) \delta 2.91-3.16$ (m, 2H, H-1"); 3.35-3.68 (m, 2H, H-5'); $3.72-$ 3.75, 3.81-3.87, 3.92-3.97 (3 m, 4H) (H-1',2',3',4'); 7.19 (s, 1H, H-4). ${ }^{13} \mathrm{C}$ NMR (75.5 MHz, $\left.\mathrm{CD}_{3} \mathrm{OD}\right) \delta 34.2\left(\mathrm{C}-1^{\prime \prime}\right) ; 63.7$ (C-5'); 72.8 (C-3'); 75.3 (C-2'); 83.8 (C-1'); 86.2 (C-4'); 106.2 (C-3); 118.0 (CN); 122.6 (C-4); 122.7 (C-5); 169.3 (C-2). $\mathrm{C}_{11} \mathrm{H}_{14} \mathrm{~N}_{2} \mathrm{O}_{4} \mathrm{~S}$ (270.30); HRMS (CI): m/z calculated for $[\mathrm{M}+\mathrm{H}]^{+}=271.07470$, found 271.07486 .

6-[(1,2-Dideoxy- $\beta$-D-ribofuranos-1-yl)methyl]thieno[2,3- $d]$ pyrimidin-4(3H)-one (18). A solution of TBAF in 1,4-dioxane $(1.0 \mathrm{M}, 1.5 \mathrm{~mL})$ was added dropwise to a solution of compound $17(525 \mathrm{mg}, 1.0 \mathrm{mmol})$ in 1,4-dioxane $(15 \mathrm{~mL})$. The reaction mixture was stirred at r.t. for $2 \mathrm{~h}$, and then concentrated. Flash chromatography (solvent $\mathrm{C}_{1}$ ) of the residue provided compound 18 (220 mg, 78\%) as colorless solid; m.p. $204{ }^{\circ} \mathrm{C} ;[\alpha]_{\mathrm{D}}^{23}+5.6(c 1.0, \mathrm{DMSO}) ; R_{f}=0.22\left(\right.$ solvent $\left.\mathrm{C}_{3}\right)$. ${ }^{1} \mathrm{H}$ NMR $\left(250 \mathrm{MHz}, \mathrm{DMSO}-d_{6}\right) \delta 1.62\left(\mathrm{ddd}, 1 \mathrm{H},{ }^{3} J_{1^{\prime}, 2^{\prime} \mathrm{a}} 9.7 \mathrm{~Hz},{ }^{3} J_{2^{\prime} \mathrm{a}, 3^{\prime}} 5.9 \mathrm{~Hz},{ }^{2} J_{2^{\prime} \mathrm{a}, 2^{\prime} \mathrm{b}} 12.7 \mathrm{~Hz}, \mathrm{H}-\right.$ $\left.2^{\prime} \mathrm{a}\right) ; 1.77$ (ddd, $\left.1 \mathrm{H},{ }^{3} J_{2^{\prime} \mathrm{b}, 3^{\prime}} 2.1 \mathrm{~Hz},{ }^{3} J_{1^{\prime}, 2^{\prime} \mathrm{b}} 5.6 \mathrm{~Hz}, \mathrm{H}-2^{\prime} \mathrm{b}\right) ; 3.02$ (m, 2H, H-1"); 3.26 (dd, $1 \mathrm{H},{ }^{3} J_{4^{\prime}, 5^{\prime} \mathrm{a}}$ $\left.6.0 \mathrm{~Hz},{ }^{2} J_{5^{\prime} \mathrm{a}, 5^{\prime} \mathrm{b}} 11.4 \mathrm{~Hz}, \mathrm{H}-5^{\prime} \mathrm{a}\right) ; 3.36$ (dd, $\left.1 \mathrm{H},{ }^{3} J_{4^{\prime}, 5^{\prime} \mathrm{b}} 4.6 \mathrm{~Hz}, \mathrm{H}-5 \mathrm{f}^{\prime} \mathrm{b}\right) ; 3.64$ (ddd, $1 \mathrm{H},{ }^{3} J_{3^{\prime}, 4^{\prime}} 2.6 \mathrm{~Hz}$, H-4'); 4.02 (m, 1H, H-3'); 4.22 (m, 1H, H-1'); 4.63 (br s, 1H, OH-5'); 4.90 (br s, 1H, OH-3'); 7.15 (s, 1H, H-5); 8.04 (s, 1H, H-2); 12.38 (br s, 1H, NH). ${ }^{13} \mathrm{C}$ NMR (62.9 MHz, DMSO- $\left.d_{6}\right) \delta 35.7$ (C-1"); 39.6 (C-2'); 62.4 (C-5'); 72.0 (C-3'); 77.4 (C-1'); 87.6 (C-4'); 119.7 (C-5); 124.5 (C-4a); 138.5 (C-6); 145.1 (C-2); 157.1 (C-7a); 163.4 (C-4). ESI-MS(+):m/z= $283[\mathrm{M}+\mathrm{H}]^{+}$. Anal. Calcd for $\mathrm{C}_{12} \mathrm{H}_{13} \mathrm{~N}_{2} \mathrm{O}_{4} \mathrm{~S}$ (282.32): C, 51.05; H 5.00; N, 9.92; S, 11.36. Found: C, 50.94; H, 4.95; N, 9.65; S, 11.16 .

\section{Preparation of pyrimidines (21-26, 31 and 32). General procedure}

$\mathrm{H}_{2} \mathrm{O}(75 \mu \mathrm{L})$ was added to a solution of compound 19 (493 mg, $\left.1.0 \mathrm{mmol}\right) ; 20$ (569 mg, 1.0 $\mathrm{mmol}), 29$ (455 mg, $1.0 \mathrm{mmol})$, or $30(517 \mathrm{mg}, 1.0 \mathrm{mmol})$ in ethyl acetate $(7.5 \mathrm{~mL})$. Sodium 
carbonate $(254 \mathrm{mg}, 2.4 \mathrm{mmol})$ and acetamidinium hydrochloride (132 mg, $1.4 \mathrm{mmol}$ ), benzamidinium hydrochloride $(219 \mathrm{mg}, 1.4 \mathrm{mmol}$ ) or $S$-methylisothiouronium sulphate $(264 \mathrm{mg}, 1.4$ mmol) was added to the solution above and the reaction mixture was heated under reflux (monitored by TLC). The reaction mixture was cooled, diluted with ethyl acetate $(50 \mathrm{~mL})$ and washed with water $(2 \times 50 \mathrm{~mL})$, dried, and concentrated. The residue was then purified by flash chromatography.

4-[2-(5-O-tert-Butyldiphenylsilyl-2,3- $O$-isopropylidene-1-deoxy- $\beta$-D-ribofuranos-1-yl)ethyl]2-methylpyrimidine (21). Reaction time $4 \mathrm{~h}$; flash chromatography solvent $\mathrm{B}_{2} ;(426 \mathrm{mg}, 80 \%)$ colorless solid; m.p. $90-92{ }^{\circ} \mathrm{C}$ (ethyl acetate $-n$-heptane); $[\alpha]_{\mathrm{D}}^{23}-10.7\left(c 1.0, \mathrm{CHCl}_{3}\right) ; R_{f}=0.21$ (solvent $\left.\mathrm{B}_{2}\right) .{ }^{1} \mathrm{H}$ NMR $\left(250 \mathrm{MHz}, \mathrm{CDCl}_{3}\right) \delta 1.04$ [s, 9H, $\left.\mathrm{C}\left(\mathrm{CH}_{3}\right)_{3}\right] ; 1.34,1.51\left[2 \mathrm{~s}, 6 \mathrm{H}, \mathrm{C}\left(\mathrm{CH}_{3}\right)_{2}\right]$; 1.90-2.13 (m, 2H, H-2"); 2.69 (s, 3H, 2- $\mathrm{CH}_{3}$ ); 2.81-2.92 (m, 2H, H-1"); 3.78 (m, 2H, H-5'); 3.88 (dt, $\left.1 \mathrm{H},{ }^{3} J_{1^{\prime}, 2^{\prime}} 5.4 \mathrm{~Hz},{ }^{3} J_{1^{\prime}, 2^{\prime \prime}} 7.8 \mathrm{~Hz}, \mathrm{H}-1^{\prime}\right) ; 4.04$ (q, $\left.1 \mathrm{H},{ }^{3} J_{3^{\prime}, 4^{\prime}} 3.7 \mathrm{~Hz},{ }^{3} J_{4^{\prime}, 5^{\prime}} 3.7 \mathrm{~Hz}, \mathrm{H}-4^{\prime}\right) ; 4.36$ (dd, $\left.1 \mathrm{H},{ }^{3} J_{2^{\prime}, 3^{\prime}} 6.7 \mathrm{~Hz}, \mathrm{H}-2^{\prime}\right) ; 4.73$ (dd, 1H, H-3'); 6.94 (d, $\left.1 \mathrm{H},{ }^{3} J_{5,6} 5.0 \mathrm{~Hz}, \mathrm{H}-5\right) ; 7.35-7.47,7.63-7.71$

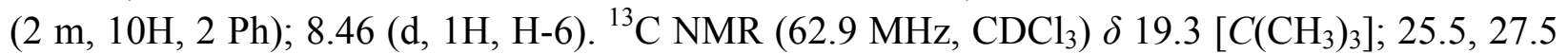
$\left[\mathrm{C}\left(\mathrm{CH}_{3}\right)_{2}\right] ; 25.8\left(2-\mathrm{CH}_{3}\right) ; 26.8\left[\mathrm{C}\left(\mathrm{CH}_{3}\right)_{3}\right] ; 32.7,33.9\left(\mathrm{C}-1^{\prime \prime}, \mathrm{C}-2^{\prime \prime}\right) ; 64.2\left(\mathrm{C}-5^{\prime}\right) ; 81.9\left(\mathrm{C}-3^{\prime}\right), 83.5$ $\left(\mathrm{C}-1^{\prime}\right) ; 84.3$ (C-4'); 84.4 (C-2'); $114.1\left[\mathrm{C}\left(\mathrm{CH}_{3}\right)_{2}\right] ; 117.4(\mathrm{C}-5) ; 127.6,127.7,129.7,129.8,135.6$, 135.7 (2 o-, $m-, p$-Ph); 133.2, 133.3 (2 i-Ph); 156.3 (C-6); 167.7 (C-2); 170.2 (C-4). $\mathrm{C}_{31} \mathrm{H}_{40} \mathrm{~N}_{2} \mathrm{O}_{4} \mathrm{Si}$ (532.74); HRMS (EI): $\mathrm{m} / \mathrm{z}$ calculated for $[\mathrm{M}]^{+}=532.27519$; found 532.27465.

4-[2-(5-O-tert-Butyldiphenylsilyl-2,3- $\boldsymbol{O}$-isopropylidene-1-deoxy- $\beta$-D-ribofuranos-1-yl)ethyl]2-methyl-6-phenylpyrimidine (22). Reaction time $24 \mathrm{~h}$; flash chromatography solvent $\mathrm{B}_{5}$; (365 mg, 60\%) colorless solid; m.p. $96-98^{\circ} \mathrm{C}$ (ethyl acetate $-n$-heptane); $[\alpha]_{\mathrm{D}}^{23}-5.4$ (c 1.0, $\mathrm{CHCl}_{3}$ ); $R_{f}=0.39$ (solvent $\left.\mathrm{B}_{4}\right) .{ }^{1} \mathrm{H} \mathrm{NMR}\left(250 \mathrm{MHz}, \mathrm{CDCl}_{3}\right) \delta 1.05\left[\mathrm{~s}, 9 \mathrm{H}, \mathrm{C}\left(\mathrm{CH}_{3}\right)_{3}\right] ; 1.34,1.50[2 \mathrm{~s}, 6 \mathrm{H}$, $\mathrm{C}\left(\mathrm{CH}_{3}\right)_{2}$ ]; 1.99-2.13 (m, 2H, H-2"); 2.76 (s, 3H, 2-CH $) ; 2.88-2.96$ (m, 2H, H-1"); 3.80 (m, 2H, H-5'); 3.93 (dt, $\left.1 \mathrm{H},{ }^{3} J_{1^{\prime}, 2^{\prime}} 5.4 \mathrm{~Hz},{ }^{3} J_{1^{\prime}, 2^{\prime \prime}} 7.5 \mathrm{~Hz}, \mathrm{H}-1^{\prime}\right)$; 4.05 (q, $1 \mathrm{H},{ }^{3} J_{3^{\prime}, 4^{\prime}} 3.7 \mathrm{~Hz},{ }^{3} J_{4^{\prime}, 5^{\prime}} 3.7 \mathrm{~Hz}, \mathrm{H}-4^{\prime}$ ); 4.39 (dd, 1H, $\left.{ }^{3} J_{2^{\prime}, 3^{\prime}} 6.7 \mathrm{~Hz}, \mathrm{H}-2^{\prime}\right) ; 4.74$ (dd, 1H H-3'); 7.33 (s, 1H, H-5); 7.36-7.48, 7.67-7.72,

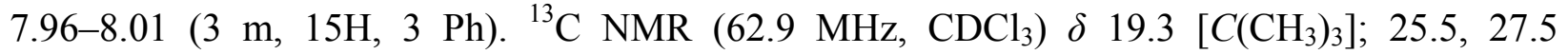

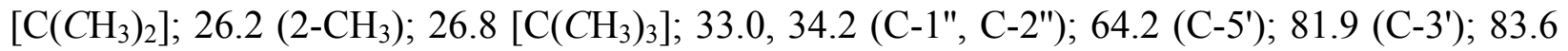
(C-1'); 84.3 (C-4'); 84.9 (C-2'); 113.0 (C-5); $114.0\left[C\left(\mathrm{CH}_{3}\right)_{2}\right]$; 127.3, 127.7, 127.8, 128.8, 129.7, 129.8, 130.5, 135.6 (3 o-, $m-, p$-Ph); 133.2, 133.3,137.3 (3 i-Ph); 164.0 (C-6); 168.1 (C-2); 170.2 (C-4). CI-MS: $m / z(\%)=609\left(100,[\mathrm{M}+\mathrm{H}]^{+}\right)$. Anal. Calcd for $\mathrm{C}_{37} \mathrm{H}_{44} \mathrm{~N}_{2} \mathrm{O}_{4} \mathrm{Si}$ (608.84): C, 72.99; H, 7.28; N, 4.60. Found: C, 72.63; H, 7.26; N, 4.33.

\section{4-[2-(5-O-tert-Butyldiphenylsilyl-2,3- $O$-isopropylidene-1-deoxy- $\beta$-D-ribofuranos-1-yl)ethyl]-}

2-phenylpyrimidine (23). Reaction time $2.5 \mathrm{~h}$; flash chromatography solvent $\mathrm{B}_{5}$; (559 mg, 94\%) colorless foam; $[\alpha]_{\mathrm{D}}^{25}-12.5\left(c 1.0, \mathrm{CHCl}_{3}\right) ; R_{f}=0.30$ (solvent $\left.\mathrm{B}_{5}\right) .{ }^{1} \mathrm{H} \mathrm{NMR}\left(250 \mathrm{MHz}, \mathrm{CDCl}_{3}\right) \delta$ $1.06\left[\mathrm{~s}, 9 \mathrm{H}, \mathrm{C}\left(\mathrm{CH}_{3}\right)_{3}\right]$; 1.36, $1.53\left[2 \mathrm{~s}, 6 \mathrm{H}, \mathrm{C}\left(\mathrm{CH}_{3}\right)_{2}\right] ; 2.08-2.28$ (m, 2H, H-2"); 2.96 (m, 2H, H$\left.1^{\prime \prime}\right) ; 3.81$ (m, 2H, H-5'); 3.97 (dt, $\left.1 \mathrm{H},{ }^{3} J_{1^{\prime}, 2^{\prime}} 5.3 \mathrm{~Hz},{ }^{3} J_{1^{\prime}, 2^{\prime \prime}} 8.0 \mathrm{~Hz}, \mathrm{H}-1^{\prime}\right) ; 4.08$ (q, $1 \mathrm{H},{ }^{3} J_{3^{\prime}, 4^{\prime}} 3.6 \mathrm{~Hz}$, $\left.{ }^{3} J_{4^{\prime}, 5^{\prime}} 3.6 \mathrm{~Hz}, \mathrm{H}-4^{\prime}\right) ; 4.40$ (dd, $\left.1 \mathrm{H},{ }^{3} J_{2^{\prime}, 3^{\prime}} 6.7 \mathrm{~Hz}, \mathrm{H}-2^{\prime}\right) ; 4.76$ (dd, 1H, H-3'); 7.01 (d, 1H, ${ }^{3} J_{5,6} 5.2$ Hz, H-5); 7.34-7.41, 7.44-7.48, 7.67-7.72, 8.43-8.47 (4 m, 15H, 3 Ph); 8.64 (d, 1H, H-6).

${ }^{13} \mathrm{C}$ NMR $\left(75.5 \mathrm{MHz}, \mathrm{CDCl}_{3}\right) \delta 19.3\left[\mathrm{C}\left(\mathrm{CH}_{3}\right)_{3}\right] ; 25.6,27.5\left[\mathrm{C}\left(\mathrm{CH}_{3}\right)_{2}\right] ; 26.8\left[\mathrm{C}\left(\mathrm{CH}_{3}\right)_{3}\right] ; 32.3$,

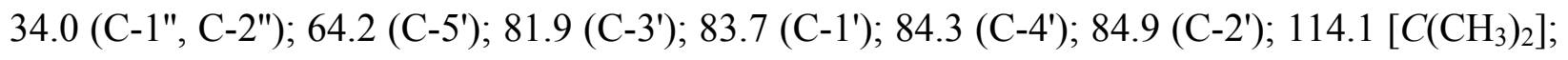


118.2 (C-5); 127.6, 127.7, 128.2, 128.5, 129.7, 129.8, 130.6, 135.6, 135.7 (3 o-, m-, p-Ph); 133.2, 133.3, 137.7 (3 i-Ph); 156.8 (C-6); 164.2 (C-2); 170.0 (C-4). CI-MS: $m / z(\%)=595\left(100,[\mathrm{M}]^{+}\right)$. Anal. Calcd for $\mathrm{C}_{36} \mathrm{H}_{42} \mathrm{~N}_{2} \mathrm{O}_{4} \mathrm{Si}$ (594.82): C, 72.69; H, 7.12; N, 4.71. Found: C, 72.39; H, 7.31; N, 4.45 .

4-[2-(5-O-tert-Butyldiphenylsilyl-2,3- $O$-isopropylidene-1-deoxy- $\beta$-D-ribofuranos-1-yl)ethyl]2,6-diphenylpyrimidine (24). Reaction time $10 \mathrm{~h}$; flash chromatography solvent $\mathrm{B}_{6}$; $(537 \mathrm{mg}$, $80 \%$ ) colorless foam; $[\alpha]_{\mathrm{D}}^{25}-13.7\left(c 0.5, \mathrm{CHCl}_{3}\right) ; R_{f}=0.44$ (solvent $\left.\mathrm{B}_{5}\right) .{ }^{1} \mathrm{H} \mathrm{NMR}(250 \mathrm{MHz}$, $\left.\mathrm{CDCl}_{3}\right) \delta 1.07\left[\mathrm{~s}, 9 \mathrm{H}, \mathrm{C}\left(\mathrm{CH}_{3}\right)_{3}\right] ; 1.37,1.53\left[2 \mathrm{~s}, 6 \mathrm{H}, \mathrm{C}\left(\mathrm{CH}_{3}\right)_{2}\right] ; 2.11-2.32$ (m, 2H, H-2"); 3.03 (m, 2H, H-1"); 3.83 (m, 2H, H-5'); 4.02 (dt, 1H, $\left.{ }^{3} J_{1^{\prime}, 2^{\prime}} 5.4 \mathrm{~Hz},{ }^{3} J_{1^{\prime}, 2^{\prime \prime}} 7.8 \mathrm{~Hz}, \mathrm{H}-1^{\prime}\right) ; 4.09$ (q, 1H, $\left.{ }^{3} J_{3^{\prime}, 4^{\prime}} 3.7 \mathrm{~Hz},{ }^{3} J_{4^{\prime}, 5^{\prime}} 3.7 \mathrm{~Hz}, \mathrm{H}-4^{\prime}\right) ; 4.44$ (dd, $\left.1 \mathrm{H},{ }^{3} J_{2^{\prime}, 3^{\prime}} 6.7 \mathrm{~Hz}, \mathrm{H}-2^{\prime}\right) ; 4.78$ (dd, 1H, H-3'); 7.36-7.41, 7.47-7.52, 7.69-7.75, 8.15-8.19, 8.59-8.63 (5 m, 20H, $4 \mathrm{Ph}) ; 7.44$ (s, 1H, H-5). ${ }^{13} \mathrm{C}$ NMR (62.9 $\left.\mathrm{MHz}, \mathrm{CDCl}_{3}\right) \delta 19.3\left[\mathrm{C}\left(\mathrm{CH}_{3}\right)_{3}\right] ; 25.6,27.5\left[\mathrm{C}\left(\mathrm{CH}_{3}\right)_{2}\right] ; 26.8\left[\mathrm{C}\left(\mathrm{CH}_{3}\right)_{3}\right] ; 32.7,34.2(\mathrm{C}-1$ ",C-2"); 64.2 (C-5'); 81.9 (C-3'); 83.8 (C-1'); 84.3 (C-4'); $85.0\left(\mathrm{C}-2^{\prime}\right) ; 113.6(\mathrm{C}-5) ; 114.1\left[C\left(\mathrm{CH}_{3}\right)_{2}\right]$; 127.2, 127.7, 127.8, 128.3, 128.4, 128.9, 129.7, 129.8, 130.5, 130.6 135.6, 135.7 (4 o-, $m-, p-\mathrm{Ph}$ ); 133.2, 133.3, 137.3, 138.1 (4 i-Ph); 163.8, 164.2 (C-2, C-6); 170.4 (C-4). CI-MS: $m / z(\%)=671$ (100, $\left.[\mathrm{M}]^{+}\right)$. Anal. Calcd for $\mathrm{C}_{42} \mathrm{H}_{46} \mathrm{~N}_{2} \mathrm{O}_{4} \mathrm{Si}$ (670.91): C, 75.19; H, 6.91; N, 4.18. Found: C, 75.25; H, 7.11; N, 3.97.

4-[2-(5-O-tert-Butyldiphenylsilyl-2,3- $O$-isopropylidene-1-deoxy- $\beta$-D-ribofuranos-1-yl)ethyl]2-methylthiopyrimidine (25). Reaction time $3 \mathrm{~h}$; flash chromatography solvent $\mathrm{B}_{6}$; $(554 \mathrm{mg}$, 98\%) colorless syrup; $[\alpha]_{\mathrm{D}}^{22}-10.5\left(c 1.0, \mathrm{CH}_{2} \mathrm{Cl}_{2}\right), R_{f}=0.45\left(\mathrm{~B}_{4}\right) .{ }^{1} \mathrm{H}$ NMR $\left(250 \mathrm{MHz}, \mathrm{CDCl}_{3}\right)$

$\delta 1.05$ [s, 9H, C(CH3) $)_{3}$; 1.34, $1.51\left[2 \mathrm{~s}, 6 \mathrm{H}, \mathrm{C}\left(\mathrm{CH}_{3}\right)_{2}\right] ; 1.93-2.14$ (m, 2H, H-1"); 2.53 (s, 3H, $\mathrm{SCH}_{3}$ ); 2.78-2.86 (m, 2H, H-2"); 3.78 (m, 2H, H-5'); 3.88 (d't', 1H, ${ }^{3} J_{1^{\prime}, 2^{\prime}} 5.4 \mathrm{~Hz},{ }^{3} J_{1^{\prime}, 2^{\prime \prime}} 8.0 \mathrm{~Hz}, \mathrm{H}-$ $\left.1^{\prime}\right) ; 4.04$ (q, $\left.1 \mathrm{H},{ }^{3} J_{3^{\prime}, 4^{\prime}} 3.7 \mathrm{~Hz},{ }^{3} J_{4^{\prime}, 5^{\prime}} 3.7 \mathrm{~Hz}, \mathrm{H}-4^{\prime}\right) ; 4.36$ (dd, $\left.1 \mathrm{H},{ }^{3} J_{2^{\prime}, 3^{\prime}} 6.7 \mathrm{~Hz}, \mathrm{H}-2^{\prime}\right) ; 4.72$ (dd, $1 \mathrm{H}$, H-3'); 6.76 (d, 1H, $\left.{ }^{3} J_{5,6} 5.0 \mathrm{~Hz}, \mathrm{H}-5\right) ; 7.33-7.42,7.66-7.71$ (2 m, 10H, $\left.2 \mathrm{Ph}\right) ; 8.34$ (d, 1H, H-6).

${ }^{13} \mathrm{C} \mathrm{NMR}\left(75.5 \mathrm{MHz}, \mathrm{CDCl}_{3}\right) \delta 14.0\left(\mathrm{SCH}_{3}\right) ; 19.3\left[\mathrm{C}\left(\mathrm{CH}_{3}\right)_{3}\right] ; 25.4,27.5\left[\mathrm{C}_{\left.\left(\mathrm{CH}_{3}\right)_{2}\right] ; 26.8}\right.$ [C( $\left.\left(\mathrm{CH}_{3}\right)_{3}\right]$; 32.2, 33.7 (C-1", C-2"); $64.2\left(\mathrm{C}-5^{\prime}\right) ; 81.9\left(\mathrm{C}-3^{\prime}\right) ; 83.5\left(\mathrm{C}-1^{\prime}\right) ; 84.2\left(\mathrm{C}-4^{\prime}\right) ; 84.9\left(\mathrm{C}-2^{\prime}\right)$; $114.1\left[\mathrm{C}\left(\mathrm{CH}_{3}\right)_{2}\right] ; 115.4(\mathrm{C}-5) ; 127.6,127.7,129.7,129.8,135.6,135.7$ (2 o-, $\left.m-, p-\mathrm{Ph}\right) ; 133.2$, 133.3 (2 i-Ph); 156.6 (C-6); 170.2 (C-4); 172.3 (C-2). $\mathrm{C}_{31} \mathrm{H}_{40} \mathrm{~N}_{2} \mathrm{O}_{4} \mathrm{SSi}$ (564.81); HRMS (CI-MS): $\mathrm{m} / \mathrm{z}$ calculated for $[\mathrm{M}+\mathrm{H}]^{+}=565.25344$; found 565.25508 .

4-[2-(5-O-tert-Butyldiphenylsilyl-2,3- $O$-isopropylidene-1-deoxy- $\beta$-D-ribofuranos-1-yl)ethyl]2-methylthio-6-phenylpyrimidine (26). Reaction time $24 \mathrm{~h}$; flash chromatography solvent $\mathrm{B}_{7}$; (513 mg, 80\%) colorless syrup; $[\alpha]_{\mathrm{D}}^{22}-8.1\left(c 1.0, \mathrm{CH}_{2} \mathrm{Cl}_{2}\right) ; R_{f}=0.51$ (solvent $\left.\mathrm{B}_{4}\right)$. ${ }^{1} \mathrm{H}$ NMR $(250$ $\left.\mathrm{MHz}, \mathrm{CDCl}_{3}\right) \delta 1.05\left[\mathrm{~s}, 9 \mathrm{H}, \mathrm{C}\left(\mathrm{CH}_{3}\right)_{3}\right] ; 1.34,1.51\left[2 \mathrm{~s}, 6 \mathrm{H}, \mathrm{C}\left(\mathrm{CH}_{3}\right)_{2}\right] ; 1.98-2.18$ (m, 2H, H-2"); $2.62\left(\mathrm{~s}, 3 \mathrm{H}, \mathrm{SCH}_{3}\right) ; 2.82-2.97$ (m, 2H, H-1"); 3.80 (m, 2H, H-5'); 3.94 (dt, $1 \mathrm{H},{ }^{3} J_{1^{\prime}, 2^{\prime}} 5.2 \mathrm{~Hz},{ }^{3} J_{1^{\prime}, 2^{\prime \prime}}$ $\left.7.6 \mathrm{~Hz}, \mathrm{H}-1^{\prime}\right)$ ); 4.05 (q, $\left.1 \mathrm{H},{ }^{3} J_{3^{\prime}, 4^{\prime}} 3.8 \mathrm{~Hz},{ }^{3} J_{4^{\prime}, 5^{\prime}} 3.8 \mathrm{~Hz}, \mathrm{H}-4^{\prime}\right) ; 4.38$ (dd, $\left.1 \mathrm{H},{ }^{3} J_{2^{\prime}, 3^{\prime}} 6.7 \mathrm{~Hz}, \mathrm{H}-2^{\prime}\right) ; 4.74$ (dd, 1H, H-3'); 7.20 (s, 1H, H-5); 7.33-7.49, 7.67-7.72, 7.99-8.04 (3 m, 15H, $3 \mathrm{Ph}) .{ }^{13} \mathrm{C} \mathrm{NMR}$ $\left(75.5 \mathrm{MHz}, \mathrm{CDCl}_{3}\right) \delta 14.1\left(\mathrm{SCH}_{3}\right) ; 19.3\left[\mathrm{C}\left(\mathrm{CH}_{3}\right)_{3}\right] ; 25.5,27.5\left[\mathrm{C}\left(\mathrm{CH}_{3}\right)_{2}\right] ; 26.8\left[\mathrm{C}\left(\mathrm{CH}_{3}\right)_{3}\right] ; 32.6$, 33.7 (C-1", C-2"); 64.2 (C-5'); 81.8 (C-3'); 83.6 (C-1'); 84.3 (C-4'); 84.9 (C-2'); 111.1 (C-5); $114.1\left[\mathrm{C}\left(\mathrm{CH}_{3}\right)_{2}\right] ; 127.2,127.6,127.7,128.8,129.7,129.8,131.0,135.6$ (3 o-, m-, p-Ph); 133.2, 133.3, 136.5 (3 i-Ph); $164.0(\mathrm{C}-6) ; 170.3(\mathrm{C}-4) ; 172.0(\mathrm{C}-2)$. CI-MS: m/z (\%) = $641\left(100,[\mathrm{M}]^{+}\right)$. 
Anal. Calcd for $\mathrm{C}_{37} \mathrm{H}_{44} \mathrm{~N}_{2} \mathrm{O}_{4} \mathrm{SSi}$ (640.91): C, 69.34; H, 6.92; N, 4.37; S, 5.00. Found: C, 69.63; H, 7.16; N, 4.12 ; S, 4.89.

\section{4-[2-[3,5-O-(Tetraisopropyldisiloxan-1,3-diyl)-1,2-dideoxy- $\beta$-D-ribofuranos-1-yl]ethyl]-2-} methylthiopyrimidine (31). Reaction time $3 \mathrm{~h}$; flash chromatography solvent $\mathrm{B}_{5}$; (503 $\mathrm{mg}$, 98\%) colorless syrup; $[\alpha]_{\mathrm{D}}^{22}-25.7\left(c 1.0, \mathrm{CH}_{2} \mathrm{Cl}_{2}\right) ; R_{f}=0.50$ (solvent $\left.\mathrm{B}_{2}\right)$. ${ }^{1} \mathrm{H}$ NMR $(250 \mathrm{MHz}$, $\left.\mathrm{CDCl}_{3}\right) \delta$ 0.99-1.05 [m, 28H, $\left.4 \mathrm{CH}\left(\mathrm{CH}_{3}\right)_{2}\right] ; 1.75-2.14$ (m, 4H, H-2", H-2'); 2.53 (s, 3H, $\left.\mathrm{SCH}_{3}\right)$; 2.75 (m, 2H, H-1"); 3.66-3.76 (m, 2H, H-5'a, H-4'); 3.92-4.02 (m, 1H, H-5'b); 4.03-4.12 (m, 1H, H-1'); 4.36 (dt, $\left.1 \mathrm{H},{ }^{3} J_{2^{\prime} \mathrm{b}, 3^{\prime}} 4.5 \mathrm{~Hz},{ }^{3} J_{2^{\prime} \mathrm{a}, 3^{\prime}} 7.9 \mathrm{~Hz},{ }^{3} J_{3^{\prime}, 4^{\prime}} 7.9 \mathrm{~Hz}, \mathrm{H}-3^{\prime}\right) ; 6.80$ (d, $1 \mathrm{H},{ }^{3} J_{5,6} 5.1 \mathrm{~Hz}, \mathrm{H}_{-}$ 5); 8.35 (d, 1H, H-6). ${ }^{13} \mathrm{C} \mathrm{NMR}\left(62.9 \mathrm{MHz}, \mathrm{CDCl}_{3}\right) \delta 12.5,12.9,13.3,13.4\left[4 \mathrm{CH}\left(\mathrm{CH}_{3}\right)_{2}\right] ; 14.0$ $\left(\mathrm{SCH}_{3}\right) ; 16.9,17.0,17.1,17.2,17.3,17.4,17.5$ (2) [4 $\left.\mathrm{CH}\left(\mathrm{CH}_{3}\right)_{2}\right] ; 33.8,33.9$ (C-1",C-2"); 40.3 (C-2'); 63.8 (C-5'); 73.6 (C-3'); 76.8 (C-1'); 85.9 (C-4'); 115.4 (C-5); 156.8 (C-6); 170.4 (C-4); 172.3 (C-2). Anal. Calcd for $\mathrm{C}_{24} \mathrm{H}_{44} \mathrm{~N}_{2} \mathrm{O}_{4} \mathrm{SSi}_{2}$ (512.85): C, 56.21; H, 8.65; N, 5.46; S, 6.25. Found: C, 56.31; H, 8.82; N, 5.22; S, 6.01.

4-[2-[3,5-O-(Tetraisopropyldisiloxan-1,3-diyl)-1,2-dideoxy- $\beta$-D-ribofuranos-1-yl]ethyl]-2methyl-6-phenylpyrimidine (32). Reaction time $24 \mathrm{~h}$; flash chromatography solvent $\mathrm{B}_{7}$; (496 mg, 89\%) colorless syrup; $[\alpha]_{\mathrm{D}}^{25}:-25.1$ (c 1.0, $\left.\mathrm{CH}_{2} \mathrm{Cl}_{2}\right) ; R_{f}=0.31$ (solvent $\left.\mathrm{B}_{7}\right) .{ }^{1} \mathrm{H}$ NMR $(250$ $\left.\mathrm{MHz}, \mathrm{CDCl}_{3}\right) \delta 0.99-1.10\left[\mathrm{~m}, 28 \mathrm{H}, 4 \mathrm{CH}\left(\mathrm{CH}_{3}\right)_{2}\right] ; 1.86\left(\mathrm{dt}, 1 \mathrm{H}^{3} J_{1^{\prime}, 2^{\prime} \mathrm{a}} 8.0 \mathrm{~Hz},{ }^{3} J_{2^{\prime} \mathrm{a}, 3^{\prime}} 8.0 \mathrm{~Hz},{ }^{2} J_{2^{\prime} \mathrm{a}, 2^{\prime} \mathrm{b}}\right.$ $\left.12.9 \mathrm{~Hz}, \mathrm{H}-2^{\prime} \mathrm{a}\right) ; 1.92-2.03$ (m, 2H, H-2"); 2.06 (ddd, 1H, ${ }^{3} J_{2^{\prime}} \mathrm{b}, 3^{\prime} 4.6 \mathrm{~Hz},{ }^{3} J_{1^{\prime}, 2^{\prime} \mathrm{b}} 6.7 \mathrm{~Hz}, \mathrm{H}-2^{\prime} \mathrm{b}$ ); 2.75 (s, 3H, 2-CH $)$ ) 2.86 (m, 2H, H-1"); 3.74 (m, 2H, H-5'a, H-4'); 4.03 (m, 1H, H-5'b); 4.11 (m, $\left.1 \mathrm{H}, \mathrm{H}-1^{\prime}\right) ; 4.39$ (dt, 1H, $\left.{ }^{3} J_{3^{\prime}, 4^{\prime}} 8.0 \mathrm{~Hz}, \mathrm{H}-3^{\prime}\right) ; 7.37$ (s, 1H, H-5); 7.46-7.50, 8.02-8.06 (2 m, 5H, $\mathrm{Ph}) .{ }^{13} \mathrm{C} \mathrm{NMR}\left(62.9 \mathrm{MHz}, \mathrm{CDCl}_{3}\right) \delta 12.5,12.9,13.4,13.5\left[4 \mathrm{CH}\left(\mathrm{CH}_{3}\right)_{2}\right] ; 16.9,17.0,17.1,17.2$, 17.3, 17.4, $17.6(2)$ [4 $\left.\mathrm{CH}\left(\mathrm{CH}_{3}\right)_{2}\right] ; 26.3\left(2-\mathrm{CH}_{3}\right) ; 34.3,34.8\left(\mathrm{C}-1^{\prime \prime}, 2 "\right) ; 40.3$ (C-2'); $63.8\left(\mathrm{C}-5^{\prime}\right)$; 73.6 (C-3'); 76.9 (C-1'); 85.9 (C-4'); 113.0 (C-5); 127.2, 128.9, 130.5 (o-, m-, p-Ph); 137.3 (i-Ph); 164.1 (C-6); $168.0(\mathrm{C}-2) ; 170.5(\mathrm{C}-4)$.

ESI-MS (+): $m / z=557[\mathrm{M}+\mathrm{H}]^{+}$. Anal. Calcd for $\mathrm{C}_{30} \mathrm{H}_{48} \mathrm{~N}_{2} \mathrm{O}_{4} \mathrm{Si}_{2}$ (556.88): C, 64.70; H, 8.69; N, 5.03. Found: C, 64.51; H, 8.76; N, 4.88.

\section{4-[2-(2,3- $O$-Isopropylidene-1-deoxy- $\beta$-D-ribofuranos-1-yl)ethyl]-2-methylthiopyrimidine}

(27). A solution of TBAF in 1,4-dioxane $(1.0 \mathrm{M}, 1.5 \mathrm{~mL})$ was added dropwise to a solution of compound 25 (565 mg, $1.0 \mathrm{mmol})$ in 1,4-dioxane $(15 \mathrm{~mL})$. The reaction mixture was stirred at r.t. for $4 \mathrm{~h}$ (monitored by TLC), and then concentrated. Flash chromatography $\left(\mathrm{B}_{1}\right)$ of the residue provided compound $27(277 \mathrm{mg}, 85 \%)$ as a colorless syrup; $[\alpha]_{\mathrm{D}}^{23}-14.6\left(c 1.0, \mathrm{CH}_{2} \mathrm{Cl}_{2}\right) ; R_{f}=$ 0.28 (solvent $\mathrm{B}_{1}$ ). ${ }^{1} \mathrm{H}$ NMR $\left(300 \mathrm{MHz}, \mathrm{CDCl}_{3}\right) \delta 1.33,1.51\left[2 \mathrm{~s}, 6 \mathrm{H}, \mathrm{C}\left(\mathrm{CH}_{3}\right)_{2}\right] ; 1.98-2.12(\mathrm{~m}$, $\left.2 \mathrm{H}, \mathrm{H}-2^{\prime \prime}\right) ; 2.54$ (s, 3H, $\left.\mathrm{SCH}_{3}\right) ; 2.72-2.89$ (m, 2H, H-1"); 3.66 (ddd, $1 \mathrm{H},{ }^{3} J_{4^{\prime}, 5^{\prime} \mathrm{a}} 4.0 \mathrm{~Hz},{ }^{3} J_{5^{\prime} \mathrm{a}, \mathrm{OH}} 7.8$ $\left.\mathrm{Hz},{ }^{2} J_{5^{\prime} \mathrm{a}, 5^{\prime} \mathrm{b}} 11.9 \mathrm{~Hz}, \mathrm{H}-5^{\prime} \mathrm{a}\right) ; 3.78$ (ddd, $1 \mathrm{H},{ }^{3} J_{5^{\prime} \mathrm{b}, \mathrm{OH}} 3.2 \mathrm{~Hz},{ }^{3} J_{4^{\prime}, 5^{\prime} \mathrm{b}} 4.0 \mathrm{~Hz}, \mathrm{H}-5{ }^{\prime} \mathrm{b}$ ); 3.90 (dt, $1 \mathrm{H}$, $\left.{ }^{3} J_{1^{\prime}, 2^{\prime}} 5.8 \mathrm{~Hz},{ }^{3} J_{1^{\prime}, 2^{\prime \prime}} 7.0 \mathrm{~Hz}, \mathrm{H}-1^{\prime}\right) ; 3.98$ (q, $\left.1 \mathrm{H},{ }^{3} J_{3^{\prime}, 4^{\prime}} 4.0 \mathrm{~Hz}, \mathrm{H}-4^{\prime}\right) ; 4.34$ (dd, $1 \mathrm{H},{ }^{3} J_{2^{\prime}, 3^{\prime}} 6.7 \mathrm{~Hz}$, H-2'); 4.64 (dd, 1H, H-3'); $6.82\left(\mathrm{~d}, 1 \mathrm{H},{ }^{3} J_{5,6} 5.0 \mathrm{~Hz}, \mathrm{H}-5\right) ; 8.37$ (d, 1H, H-6); OH signal not detected. ${ }^{13} \mathrm{C}$ NMR (75.5 MHz, $\left.\mathrm{CDCl}_{3}\right) \delta 14.1\left(\mathrm{SCH}_{3}\right) ; 25.4,27.5\left[\mathrm{C}\left(\mathrm{CH}_{3}\right)_{2}\right] ; 31.8,33.5(\mathrm{C}-1$ ", C2"); 62.7 (C-5'); 81.5 (C-3'); 83.8 (C-1'); 84.3 (C-4'); 84.8 (C-2'); 114.6 [C( $\left.\left.\mathrm{CH}_{3}\right)_{2}\right]$; 115.5 (C-5); 156.9 (C-6); 170.1 (C-4); 172.4 (C-2). CI-MS: $m / z(\%)=327\left(100,[\mathrm{M}+\mathrm{H}]^{+}\right) . \mathrm{C}_{15} \mathrm{H}_{22} \mathrm{~N}_{2} \mathrm{O}_{4} \mathrm{~S}$ (326.41): C, 55.19; H, 6.79; N, 8.58; S, 9.82. Found: C, 55.09, H, 7.07; N, 8.36; S, 9.58. 
4-[2-(2,3-O-Isopropylidene-1-deoxy- $\beta$-D-ribofuranos-1-yl)ethyl]-2-methylthiopyrimidine (28). $\mathrm{Aq} \mathrm{HCl}(0.1 \mathrm{M}, 2.5 \mathrm{~mL})$ was added to a solution of compound 25 (565 mg, $1.0 \mathrm{mmol})$ in EtOH $(8 \mathrm{~mL})$, and the mixture was stirred overnight at r.t (monitored by TLC). The mixture was then neutralized by addition of solid $\mathrm{NaHCO}_{3}$, and concentrated after addition of a small amount of silica gel. The residue was purified by flash chromatography (solvent $\mathrm{C}_{4}$ ) to afford compound $28(74 \%)$ as a colorless syrup; $[\alpha]_{\mathrm{D}}^{23}-24.3(c 0.5, \mathrm{MeOH}) ; \mathrm{Rf}=0.32$ (solvent $\left.\mathrm{C}_{4}\right) .{ }^{1} \mathrm{H}$ NMR $(250$ MHz, DMSO- $\left.d_{6}\right) \delta 1.68-2.01$ (m, 2H, H-2"); 2.49 (s, 3H, $\left.\mathrm{SCH}_{3}\right) ; 2.65-2.85$ (m, 2H, H-1"); 3.363.46 (m, 2H, H-5'); 3.56 (m, 2H, H-1', H-2'); 3.62 (m, 1H, H-4'); 3.76 (m, 1H, H-3'); 4.63 (t, 1H, $\left.{ }^{3} J_{5^{\prime}, \mathrm{OH}} 5.6 \mathrm{~Hz}, \mathrm{OH}-5^{\prime}\right) ; 4.72$ (m, 2H, OH-2', OH-3'); 7.08 (d, 1H, $\left.{ }^{3} J_{5,6} 5.1 \mathrm{~Hz}, \mathrm{H}-5\right) ; 8.48(\mathrm{~d}, 1 \mathrm{H}$, H-6). ${ }^{13} \mathrm{C}$ NMR (75.5 MHz, DMSO- $\left.d_{6}\right) \delta 13.4\left(\mathrm{SCH}_{3}\right) ; 31.7$ (C-2"); 33.2 (C-1"); 62.0 (C-5'); 71.3 (C-3'); 74.4 (C-2'); 80.9 (C-1'); 84.6 (C-4'); 116.0 (C-5); 157.2 (C-6); 170.6, 170.9 (C-2,4).

$\mathrm{C}_{12} \mathrm{H}_{19} \mathrm{~N}_{2} \mathrm{O}_{4} \mathrm{~S}$ (286.35); HRMS (CI-MS): $\mathrm{m} / \mathrm{z}$ calculated for $[\mathrm{M}+\mathrm{H}]^{+}=287.10600$; found 287.10513

4-[2-(1,2-Dideoxy- $\beta$-D-ribofuranos-1-yl)ethyl]-2-methylthiopyrimidine (33). Starting from compound 31 (513 mg, $1.0 \mathrm{mmol})$, compound 33 (211 mg, 78\%) was obtained after a reaction time of $24 \mathrm{~h}$ and purification by flash chromatography (solvent $\mathrm{C}_{4}$ ) as colorless a solid according to the procedure described for compound 18; m.p. $112-114{ }^{\circ} \mathrm{C} ;[\alpha]_{\mathrm{D}}^{23}-2.0(c 1.0, \mathrm{MeOH}) ; R_{f}=$ 0.36 (solvent $\mathrm{C}_{3}$ ). ${ }^{1} \mathrm{H}$ NMR $\left(250 \mathrm{MHz}, \mathrm{DMSO}-d_{6}\right) \delta 1.56$ (ddd, $1 \mathrm{H},{ }^{3} J_{2^{\prime} \mathrm{a}, 3^{\prime}} 6.0 \mathrm{~Hz},{ }^{3} J_{1^{\prime}, 2^{\prime} \mathrm{a}} 9.8 \mathrm{~Hz}$, $\left.{ }^{2} J_{2^{\prime} \mathrm{a}, 2^{\prime} \mathrm{b}} 12.7 \mathrm{~Hz}, \mathrm{H}-2^{\prime} \mathrm{a}\right) ; 1.84$ (m, 2H, H-2"); 2.02 (ddd, $1 \mathrm{H},{ }^{3} J_{2^{\prime} \mathrm{b}, 3^{\prime}} 1.6 \mathrm{~Hz},{ }^{3} J_{1^{\prime}, 2^{\prime} \mathrm{b}} 5.4 \mathrm{~Hz}, \mathrm{H}-2 \mathrm{z}^{\prime} \mathrm{b}$ ); 2.48 (s, 3H, $\mathrm{SCH}_{3}$ ); 2.72 (m, 2H, H-1"); 3.27-3.35 (m, 2H, H-5'a, H-4'); 3.59 (ddd, 1H, ${ }^{3} J_{4^{\prime}, 5^{\prime} \mathrm{b}} 2.6$ $\left.\mathrm{Hz},{ }^{3} J_{5^{\prime} \mathrm{b}, \mathrm{OH}} 5.3 \mathrm{~Hz},{ }^{2} J_{5^{\prime} \mathrm{a}, 5^{\prime} \mathrm{b}} 7.8 \mathrm{~Hz}, \mathrm{H}-5^{\prime} \mathrm{b}\right) ; 3.97$ (m, 1H, H-1'); 4.03 (m, 1H, H-3'); 7.09 (d, 1H, $\left.{ }^{3} J_{5,6} 5.1 \mathrm{~Hz}, \mathrm{H}-5\right) ; 8.49$ (d, $\left.1 \mathrm{H}, \mathrm{H}-6\right) .{ }^{13} \mathrm{C} \mathrm{NMR}\left(62.9 \mathrm{MHz}, \mathrm{DMSO}-d_{6}\right) \delta 13.4\left(\mathrm{SCH}_{3}\right) ; 33.4,33.6$ (C-1",2"); 40.2 (C-2'); 62.5 (C-5'); 72.1 (C-3'); 76.8 (C-1'); 87.3 (C-4'); 115.9 (C-5); 157.2 (C-6); 170.6, $170.9(\mathrm{C}-2,4)$. ESI-MS (+): $m / z: 271[\mathrm{M}+\mathrm{H}]^{+}$. Anal. Calcd for $\mathrm{C}_{12} \mathrm{H}_{18} \mathrm{~N}_{2} \mathrm{O}_{3} \mathrm{~S}$ (270.35): C, 53.31; H, 6.71; N, 10.36; S, 11.86. Found: C, 53.08; H, 6.50; N, 10.32; S, 11.69.

\section{Preparation of aminotriazoles (34 and 38). General procedure}

A mixture of compound 19 (246 mg, $0.5 \mathrm{mmol})$ or 29 (227 mg, $0.5 \mathrm{mmol})$ and 4H-1,2,4-triazol3-amine $(50 \mathrm{mg}, 0.6 \mathrm{mmol})$ in dry EtOH $(5 \mathrm{~mL})$ was heated under reflux for $4 \mathrm{~h}$ (monitored by TLC), and concentrated. The residue was purified by flash chromatography.

(E)-1-(5-Amino-1H-1,2,4-triazol-1-yl)-5-(5-O-tert-butyldiphenylsilyl-2,3-O-isopropylidene1-deoxy- $\boldsymbol{\beta}$-D-ribofuranos-1-yl)pent-1-en-3-one (34). Flash chromatography solvent A; (257 mg, 89\%); yellow solid; m.p. $118-119^{\circ} \mathrm{C} ;[\alpha]_{\mathrm{D}}^{23}-2.6\left(c 1.0, \mathrm{CH}_{2} \mathrm{Cl}_{2}\right) ; R_{f}=0.25$ (solvent A).

${ }^{1} \mathrm{H}$ NMR $\left(250 \mathrm{MHz}, \mathrm{CDCl}_{3}\right) \delta 1.04\left[\mathrm{~s}, 9 \mathrm{H}, \mathrm{C}\left(\mathrm{CH}_{3}\right)_{3}\right] ; 1.35,1.52\left[2 \mathrm{~s}, 6 \mathrm{H}, \mathrm{C}\left(\mathrm{CH}_{3}\right)_{2}\right] ; 1.74-1.97$ (m, 2H, H-1); 2.76 (m, 2H, H-2); 3.76 (m, 2H, H-5'); 3.82 (m, 1H, H-1'); 4.01 (q, 1H, ${ }^{3} J_{3^{\prime}, 4^{\prime}} 3.6$ $\left.\mathrm{Hz},{ }^{3} J_{4^{\prime}, 5^{\prime}} 3.6 \mathrm{~Hz}, \mathrm{H}-4^{\prime}\right) ; 4.29$ (dd, $\left.1 \mathrm{H},{ }^{3} J_{1^{\prime}, 2^{\prime}} 5.5 \mathrm{~Hz},{ }^{3} J_{2^{\prime}, 3^{\prime}} 6.7 \mathrm{~Hz}, \mathrm{H}-2^{\prime}\right) ; 4.70$ (dd, $\left.1 \mathrm{H}, \mathrm{H}-3^{\prime}\right) ; 5.81$ (br s, $2 \mathrm{H}, \mathrm{NH}_{2}$ ); 6.74 (d, 1H, $\left.{ }^{3} J_{4,5} 13.3 \mathrm{~Hz}, \mathrm{H}-4\right) ; 7.32-7.42,7.64-7.69$ (2 m, 10H, $\left.2 \mathrm{Ph}\right) ; 7.57$ (s,

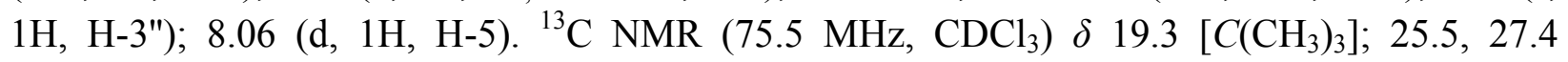
$\left[\mathrm{C}\left(\mathrm{CH}_{3}\right)_{2}\right] ; 26.8\left[\mathrm{C}\left(\mathrm{CH}_{3}\right)_{3}\right] ; 27.4(\mathrm{C}-1) ; 39.1(\mathrm{C}-2) ; 64.1\left(\mathrm{C}-5^{\prime}\right) ; 82.0\left(\mathrm{C}-3^{\prime}\right) ; 83.2\left(\mathrm{C}-1^{\prime}\right) ; 84.2$ $\left(\mathrm{C}-4{ }^{\prime}\right) ; 84.7\left(\mathrm{C}-2^{\prime}\right) ; 113.2(\mathrm{C}-4) ; 114.5\left[\mathrm{C}_{\left.\left(\mathrm{CH}_{3}\right)_{2}\right]}\right.$; 127.6, 127.7, 129.7, 129.8, 135.6, 135.7 (2 
$o$-, m-, p- Ph); 132.1 (C-5); 133.2, 133.3 (2 i-Ph); 151.7 (C-3"); 199.6 (C-3); C-5" signal not detected. $\mathrm{C}_{31} \mathrm{H}_{40} \mathrm{~N}_{4} \mathrm{O}_{5} \mathrm{Si}$ (576.76); HRMS (CI-MS): $\mathrm{m} / \mathrm{z}$ calculated for $[\mathrm{M}+\mathrm{H}]^{+}=577.28462$; found 577.28429 .

(E)-1-(5-Amino-1H-1,2,4-triazol-1-yl)-5-[(3,5-O-(tetraisopropyldisiloxan-1,3-diyl)-1,2-

dideoxy - $\beta$-D-ribofuranos-1-yl]pent-1-en-3-one (38). Flash chromatography solvent $\mathrm{B}_{1}$; $(152$ mg, 58\%) yellow solid; m.p. $170^{\circ} \mathrm{C} ;[\alpha]_{\mathrm{D}}^{25}-34.2\left(c 0.8, \mathrm{CH}_{2} \mathrm{Cl}_{2}\right) ; R_{f}=0.19$ (solvent $\mathrm{B}_{1}$ ).

${ }^{1} \mathrm{H}$ NMR $\left(250 \mathrm{MHz}, \mathrm{CDCl}_{3}\right) \delta$ 0.99-1.05 [m, 28H, $\left.4 \mathrm{CH}\left(\mathrm{CH}_{3}\right)_{2}\right]$; 1.72-1.87 (m, 3H, H-1, H-2'a); 2.02 (ddd, $1 \mathrm{H},{ }^{3} J_{2^{\prime} \mathrm{b}, 3^{\prime}} 4.8 \mathrm{~Hz},{ }^{3} J_{1^{\prime}, 2^{\prime} \mathrm{b}} 6.8 \mathrm{~Hz},{ }^{2} J_{2^{\prime} \mathrm{a}, 2^{\prime} \mathrm{b}} 12.5 \mathrm{~Hz}, \mathrm{H}-2 \mathrm{~b}$ ); 2.68 (m, 2H, H-2); 3.66-3.76 (m, 2H, H-5'a, H-4'); 3.99 (m, 1H, H-5'b); 4.04 (m, 1H, H-1'); 4.35 (dt, 1H, ${ }^{3} J_{2^{\prime} \mathrm{a}, 3^{\prime}} 8.0 \mathrm{~Hz},{ }^{3} J_{3^{\prime}, 4^{\prime}}$ 8.0 Hz, H-3'); 6.11 (br s, 2H, NH ); 6.72 (d, 1H, $\left.{ }^{3} J_{4,5} 13.3 \mathrm{~Hz}, \mathrm{H}-4\right) ; 7.56$ (s, 1H, H-3"); 8.05 (d, $1 \mathrm{H}, \mathrm{H}-5) .{ }^{13} \mathrm{C}$ NMR $\left(62.9 \mathrm{MHz}, \mathrm{CDCl}_{3}\right) \delta 12.5,12.9,13.3,13.4\left[4 \mathrm{CH}\left(\mathrm{CH}_{3}\right)_{2}\right] ; 16.9,17.0,17.1$, 17.2, 17.3, 17.4, 17.5 (2) [4 $\left.\mathrm{CH}\left(\mathrm{CH}_{3}\right)_{2}\right] ; 29.8$ (C-1); 39.3 (C-2); 40.0 (C-2'); 63.5 (C-5'); 73.2 (C-3'); 76.5 (C-1'); 85.8 (C-4'); 113.0 (C-4); 132.4 (C-5); 151.6 (C-3"); 200.1 (C-3); C-5" signal not detected. ESI-MS (-): $m / z=523[\mathrm{M}-\mathrm{H}]^{-}$. Anal. Calcd for $\mathrm{C}_{24} \mathrm{H}_{44} \mathrm{~N}_{4} \mathrm{O}_{5} \mathrm{Si}_{2}$ (524.80): C, 54.93; H, 8.45; N, 10.68. Found: C, 54.79; H, 8.15; N, 10.72.

\section{Preparation of triazolopyrimidines (35 and 39). General procedure}

Ethanolic NaOMe (1 M, $1.4 \mathrm{~mL})$ was added to a solution of compound 34 (288 $\mathrm{mg}, 0.5 \mathrm{mmol})$ or $38(262 \mathrm{mg}, 0.5 \mathrm{mmol})$ in dry EtOH $(10 \mathrm{~mL})$. After stirring at r.t. for $1 \mathrm{~h}$, the reaction mixture was neutralized with IR $120\left(\mathrm{H}^{+}\right)$Amberlite resin, filtered, dried, and concentrated. The residue was purified by flash chromatography (solvent $\mathrm{B}_{2}$ ).

5-[2-(5-O-tert-Butyldiphenylsilyl-2,3- $\boldsymbol{O}$-isopropylidene-1-deoxy- $\boldsymbol{\beta}$-D-ribofuranos-1-yl)ethyl]$[1,2,4]$ triazolo[1,5-a]pyrimidine (35). Yield (173 mg, 62\%); colorless syrup; $[\alpha]_{\mathrm{D}}^{22}-15.4(c)$,

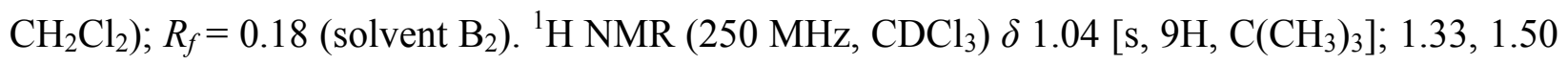
[2 s, 6H, C( $\left.\left.\mathrm{CH}_{3}\right)_{2}\right] ; 2.04-2.29$ (m, 2H, H-2"); 3.07 (m, 2H, H-1"); 3.79 (m, 2H, H-5'); 3.93 (dt, $\left.1 \mathrm{H},{ }^{3} J_{1^{\prime}, 2^{\prime}} 5.1 \mathrm{~Hz},{ }^{3} J_{1^{\prime}, 2^{\prime \prime}} 8.3 \mathrm{~Hz}, \mathrm{H}-1^{\prime}\right) ; 4.04$ (q, $\left.1 \mathrm{H},{ }^{3} J_{3^{\prime}, 4^{\prime}} 3.8 \mathrm{~Hz},{ }^{3} J_{4^{\prime}, 5^{\prime}} 3.8 \mathrm{~Hz}, \mathrm{H}-4^{\prime}\right) ; 4.38$ (dd, $1 \mathrm{H}$, $\left.{ }^{3} J_{2^{\prime}, 3^{\prime}} 6.6 \mathrm{~Hz}, \mathrm{H}-2^{\prime}\right) ; 4.72$ (dd, 1H, H-3'); 6.89 (d, 1H, $\left.{ }^{3} J_{6,7} 7.0 \mathrm{~Hz}, \mathrm{H}-6\right) ; 7.32-7.42,7.65-7.70$ (2 m, 10H, $2 \mathrm{Ph}) ; 8.43$ (s, 1H, H-2); 8.63 (d, 1H, H-7). ${ }^{13} \mathrm{C}$ NMR (75.5 MHz, CDCl $) \delta 19.3$ $\left[\mathrm{C}\left(\mathrm{CH}_{3}\right)_{3}\right] ; 25.5,27.4\left[\mathrm{C}\left(\mathrm{CH}_{3}\right)_{2}\right] ; 26.8\left[\mathrm{C}\left(\mathrm{CH}_{3}\right)_{3}\right] ; 32.1,34.6\left(\mathrm{C}-1^{\prime \prime}, \mathrm{C}-2 "\right) ; 64.1\left(\mathrm{C}-5^{\prime}\right) ; 81.8(\mathrm{C}-$ $\left.3^{\prime}\right) ; 83.4$ (C-1'); 84.3 (C-4'); 84.8 (C-2'); 110.9 (C-6); $114.1\left[\mathrm{C}\left(\mathrm{CH}_{3}\right)_{2}\right] ; 127.6,127.7,129.7$, 129.8, 135.5, 135.6 (2 o-, $m-, p-\mathrm{Ph}) ; 133.2,133.3$ (2 i-Ph); 134.9 (C-7); 155.1 (C-3a); 156.1 (C2); 168.4 (C-5). Anal. Calcd for $\mathrm{C}_{31} \mathrm{H}_{38} \mathrm{~N}_{4} \mathrm{O}_{4} \mathrm{Si}$ (558.74): C, 66.64; H, 6.85; N, 10.03. Found: C, $66.56 ; \mathrm{H}, 6.72 ; \mathrm{N}, 10.26$.

5-[2-[3,5-O-(Tetraisopropyldisiloxan-1,3-diyl)-1,2-dideoxy- $\beta$-D-ribofuranos-1-yl]ethyl][1,2,4]triazolo[1,5-a]pyrimidine (39). Yield (175 mg, 69\%); colorless solid; m.p. 80-82 ${ }^{\circ} \mathrm{C}$; $[\alpha]_{\mathrm{D}}^{25}-31.6\left(c 1.0, \mathrm{CH}_{2} \mathrm{Cl}_{2}\right) ; R_{f}=0.13$ (solvent $\left.\mathrm{B}_{2}\right) .{ }^{1} \mathrm{H}$ NMR $\left(250 \mathrm{MHz}, \mathrm{CDCl}_{3}\right) \delta 1.00-1.06[\mathrm{~m}$, $\left.28 \mathrm{H}, 4 \mathrm{CH}\left(\mathrm{CH}_{3}\right)_{2}\right] ; 1.86\left(\mathrm{dt}, 1 \mathrm{H},{ }^{3} J_{2^{\prime} \mathrm{a}, 1^{\prime}} 7.7 \mathrm{~Hz},{ }^{3} J_{2^{\prime} \mathrm{a}, 3^{\prime}} 7.7 \mathrm{~Hz},{ }^{2} J_{2^{\prime} \mathrm{a}, 2^{\prime} \mathrm{b}} 12.5 \mathrm{~Hz}, \mathrm{H}-2^{\prime} \mathrm{a}\right) ; 2.07$ (ddd, $\left.1 \mathrm{H},{ }^{3} J_{2^{\prime} \mathrm{b}, 3^{\prime}} 4.6 \mathrm{~Hz},{ }^{3} J_{1^{\prime}, 2^{\prime} \mathrm{b}} 6.7 \mathrm{~Hz},{ }^{2} J_{2^{\prime} \mathrm{a}, 2^{\prime} \mathrm{b}} 12.5 \mathrm{~Hz}, \mathrm{H}-2^{\prime} \mathrm{b}\right) ; 1.94-2.21$ (m, 2H, H-1"); 3.05 (m, 2H, H-2"); 3.66-3.77 (m, 2H, H-5'a, H-4'); 4.00 (m, 1H, H-5'b); 4.07-4.18 (m, 1H, H-1'); 4.38 (d't', $\left.1 \mathrm{H},{ }^{3} J_{2^{\prime}, 3^{\prime}} 8.0 \mathrm{~Hz},{ }^{3} J_{3^{\prime}, 4^{\prime}} 8.0 \mathrm{~Hz}, \mathrm{H}-3^{\prime}\right) ; 6.99$ (d, $\left.1 \mathrm{H},{ }^{3} J_{6,7} 7.0 \mathrm{~Hz}, \mathrm{H}-6\right) ; 8.43$ (s, 1H, H-2); 8.68 (d, 
$1 \mathrm{H}, \mathrm{H}-7) .{ }^{13} \mathrm{C} \mathrm{NMR}\left(62.9 \mathrm{MHz}, \mathrm{CDCl}_{3}\right) \delta 12.5,12.9,13.3,13.5\left[4 \mathrm{CH}\left(\mathrm{CH}_{3}\right)_{2}\right] ; 16.9,17.0,17.1$, 17.3, 17.4, 17.4, 17.4, 17.5 [4 $\left.\mathrm{CH}\left(\mathrm{CH}_{3}\right)_{2}\right] ; 34.0,34.7$ (C-1", C-2"); 40.3 (C-2'); 63.7 (C-5'); 73.4 (C-3'); 76.5 (C-1'); 86.0 (C-4'); 111.0 (C-6); 134.9 (C-7); 156.1 (C-2); 156.1 (C-3a); 168.8 (C-5). ESI-MS (-): $m / z=505[\mathrm{M}-\mathrm{H}]^{-}$. Anal. Calcd for $\mathrm{C}_{24} \mathrm{H}_{42} \mathrm{~N}_{4} \mathrm{O}_{4} \mathrm{Si}_{2}(506.79): \mathrm{C}, 56.88 ; \mathrm{H}, 8.35 ; \mathrm{N}$, 11.06. Found: C, 56.77; H, 8.15; N, 10.82 .

\section{Preparation of pyrimidobenzimidazoles (36, 37 and 40). General procedure}

A mixture of compound 19 (493 mg, $1.0 \mathrm{mmol}), 20$ (569 mg, $1.0 \mathrm{mmol})$ or 29 (455 mg, 1.0 $\mathrm{mmol})$ and $1 H$-benzo $[d]$ imidazole-2-amine $(266 \mathrm{mg}, 2.0 \mathrm{mmol})$ in dry EtOH $(10 \mathrm{~mL})$ was heated under reflux for $2 \mathrm{~h}$ ( monitored by TLC). The reaction mixture was cooled to r.t. and ethanolic $\mathrm{NaOMe}(1.0 \mathrm{M}, 3.0 \mathrm{~mL})$ was added. After stirring at r.t. for $1 \mathrm{~h}$, the reaction mixture was neutralized with IR $120\left(\mathrm{H}^{+}\right)$Amberlite resin, filtered, dried, and concentrated. The residue was purified by flash chromatography.

2-[2-(5-O-tert-Butyldiphenylsilyl-2,3- $O$-isopropylidene-1-deoxy- $\beta$-D-ribofuranos-1-yl)ethyl]pyrimido[1,2-a]benzimidazole (36). Flash chromatography solvent $\mathrm{B}_{2}$; (462 mg, 76\%) yellow foam; $[\alpha]_{\mathrm{D}}^{24}-21.3\left(c 1.0, \mathrm{CH}_{2} \mathrm{Cl}_{2}\right) ; R_{f}=0.14$ (solvent $\left.\mathrm{B}_{2}\right) .{ }^{1} \mathrm{H} \mathrm{NMR}\left(250 \mathrm{MHz}, \mathrm{CDCl}_{3}\right) \delta 1.05[\mathrm{~s}$, $\left.9 \mathrm{H}, \mathrm{C}\left(\mathrm{CH}_{3}\right)_{3}\right] ; 1.34,1.51$ [2 s, 6H, C(CH$\left.)_{2}\right] ; 2.07-2.30$ (m, 2H, H-2"); 3.06 (m, 2H, H-1"); 3.80 (m, 2H, H-5'); 3.96 (dt, $\left.1 \mathrm{H},{ }^{3} J_{1^{\prime}, 2^{\prime}} 5.0 \mathrm{~Hz},{ }^{3} J_{1^{\prime}, 2^{\prime \prime}} 8.0 \mathrm{~Hz}, \mathrm{H}-1^{\prime}\right) ; 4.04$ (q, $1 \mathrm{H},{ }^{3} J_{3^{\prime}, 4^{\prime}} 3.8 \mathrm{~Hz},{ }^{3} J_{4^{\prime}, 5^{\prime}} 3.8$ Hz, H-4'); 4.41 (dd, 1H, $\left.{ }^{3} J_{2^{\prime}, 3^{\prime}} 6.6 \mathrm{~Hz}, \mathrm{H}-2^{\prime}\right) ; 4.74$ (dd, 1H, H-3'); 6.77 (d, 1H, ${ }^{3} J_{3,4} 7.0, \mathrm{H}-3$ ); $7.33-$ 7.42, 7.66-7.71 (2 m, 11H, 2 Ph, H-7); 7.54 (m, 1H, H-8); 7.83 (d, 1H, $\left.{ }^{3} J_{6,7} 8.0 \mathrm{~Hz}, \mathrm{H}-6\right) ; 8.01$ (d, $\left.1 \mathrm{H},{ }^{3} J_{8,9} 8.2 \mathrm{~Hz}, \mathrm{H}-9\right) ; 8.59$ (d, 1H, H-4). $\left.{ }^{13} \mathrm{C} \mathrm{NMR} \mathrm{(62.9} \mathrm{MHz,} \mathrm{CDCl}_{3}\right) \delta 19.3\left[C\left(\mathrm{CH}_{3}\right)_{3}\right]$; 25.5, $27.5\left[2 \mathrm{C}\left(\mathrm{CH}_{3}\right)_{2}\right] ; 26.8\left[\mathrm{C}\left(\mathrm{CH}_{3}\right)_{3}\right] ; 31.8\left(\mathrm{C}-1^{\prime \prime}\right) ; 35.1$ (C-2"); 64.2 (C-5'); 81.8 (C-3'), 83.5 $\left(\mathrm{C}-1^{\prime}\right) ; 84.3$ (C-4'); 84.9 (C-2'); $107.8(\mathrm{C}-3) ; 110.4(\mathrm{C}-6) ; 114.1\left[\mathrm{C}\left(\mathrm{CH}_{3}\right)_{2}\right] ; 120.1$ (C-9); 121.9 (C-7); 126.4 (C-8); 126.6 (C-5a); 127.6, 127.7, 129.7, 129.8, 135.5, 135.6 (2 o-, m-, p-Ph); 132.5 (C-4); 133.3, 133.4 (2 i-Ph); 143.3 (C-9a); 150.4 (C-10a); 169.3 (C-2). $\mathrm{C}_{36} \mathrm{H}_{41} \mathrm{~N}_{3} \mathrm{O}_{4} \mathrm{Si}(607.81)$; HRMS (EI-MS): $\mathrm{m} / \mathrm{z}$ calculated for $[\mathrm{M}]^{+}=607.28663$; found 607.28608 .

2-[2-(5-O-tert-Butyldiphenylsilyl-2,3- $O$-isopropylidene-1-deoxy- $\beta$-D-ribofuranos-1-yl)ethyl]4-phenyl- pyrimido[1,2-a]benzimidazole (37). Flash chromatography solvent $\mathrm{B}_{2}$; (547 mg, $80 \%$ ) yellow foam; $[\alpha]_{\mathrm{D}}^{24}-20.2\left(c 1.0, \mathrm{CH}_{2} \mathrm{Cl}_{2}\right) ; R_{f}=0.21$ (solvent $\left.\mathrm{B}_{2}\right) .{ }^{1} \mathrm{H}$ NMR $(250 \mathrm{MHz}$, $\left.\mathrm{CDCl}_{3}\right) \delta 1.03\left[\mathrm{~s}, 9 \mathrm{H}, \mathrm{C}\left(\mathrm{CH}_{3}\right)_{3}\right] ; 1.34,1.51\left[2 \mathrm{~s}, 6 \mathrm{H}, \mathrm{C}\left(\mathrm{CH}_{3}\right)_{2}\right] ; 2.10-2.36(\mathrm{~m}, 2 \mathrm{H}, \mathrm{H}-2 ") ; 3.07$ (m, 2H, H-1"); 3.80 (m, 2H, H-5'); 4.01 (dt, $\left.1 \mathrm{H},{ }^{3} J_{1^{\prime}, 2^{\prime}} 5.0 \mathrm{~Hz},{ }^{3} J_{1^{\prime}, 2^{\prime \prime}} 8.1 \mathrm{~Hz}, \mathrm{H}-1^{\prime}\right) ; 4.05$ (q, 1H, $\left.{ }^{3} J_{3^{\prime}, 4^{\prime}} 3.8 \mathrm{~Hz},{ }^{3} J_{4^{\prime}, 5^{\prime}} 3.8 \mathrm{~Hz}, \mathrm{H}-4^{\prime}\right) ; 4.42$ (dd, $\left.1 \mathrm{H},{ }^{3} J_{2^{\prime}, 3^{\prime}} 6.7 \mathrm{~Hz}, \mathrm{H}-2^{\prime}\right) ; 4.74$ (dd, 1H, H-3'); 6.58 (s, $1 \mathrm{H}, \mathrm{H}-3) ; 6.64$ (d, 1H, $\left.{ }^{3} J_{6,7} 8.4 \mathrm{~Hz}, \mathrm{H}-6\right) ; 6.99$ (m, 1H, H-7); 7.30-7.38, 7.49-7.70 (2 m, 15H, 3 $\mathrm{Ph}) ; 7.44$ (m, 1H, H-8); 7.94 (d, 1H, $\left.{ }^{3} J_{8,9} 8.2 \mathrm{~Hz}, \mathrm{H}-9\right) .{ }^{13} \mathrm{C}$ NMR (62.9 MHz, $\left.\mathrm{CDCl}_{3}\right) \delta 19.2$ $\left[\mathrm{C}\left(\mathrm{CH}_{3}\right)_{3}\right] ; 25.5,27.5\left[\mathrm{C}\left(\mathrm{CH}_{3}\right)_{2}\right] ; 26.8\left[\mathrm{C}\left(\mathrm{CH}_{3}\right)_{3}\right] ; 31.9(\mathrm{C}-1 ") ; 34.8(\mathrm{C}-2 ") ; 64.2(\mathrm{C}-5$ '); $81.8(\mathrm{C}-$ 3'); 83.6 (C-1'); 84.3 (C-4'); 84.9 (C-2'); 108.7 (C-3); 114.1 [C( $\left.\left(\mathrm{CH}_{3}\right)_{2}\right] ; 114.4$ (C-6); 120.1 (C-9); 120.9 (C-7); 125.7 (C-8); 127.3 (C-5a); 127.6, 127.7, 128.2, 129.6, 129.7, 130.9, 135.6 (3 o-, m-, $p$-Ph); 132.2, 133.2, 133.3 (3 i-Ph); 144.6 (C-9a); 148.5 (C-4); 151.9 (C-10a); 168.1 (C-2).

$\mathrm{C}_{42} \mathrm{H}_{45} \mathrm{~N}_{3} \mathrm{O}_{4} \mathrm{Si}$ (683.91); HRMS (EI-MS): $\mathrm{m} / \mathrm{z}$ calculated for $[\mathrm{M}]^{+}=683.31793$; found 683.31738 . 


\section{2-[2-[3,5-O-(Tetraisopropyldisiloxan-1,3-diyl)-1,2-didesoxy- $\beta$-D-ribofuranos-1-yl]ethyl]-}

pyrimido[1,2-a]benzimidazole (40). Flash chromatography solvent $\mathrm{B}_{1}$; (350 $\left.\mathrm{mg}, 63 \%\right)$ colorless syrup; $[\alpha]_{\mathrm{D}}^{22}-25.7\left(c\right.$ 1.0, $\left.\mathrm{CH}_{2} \mathrm{Cl}_{2}\right) ; R_{f}=0.14$ (solvent $\left.\mathrm{B}_{2}\right) .{ }^{1} \mathrm{H}$ NMR $\left(250 \mathrm{MHz}, \mathrm{CDCl}_{3}\right) \delta 1.00$ 1.07 [m, 28H, $\left.4 \mathrm{CH}\left(\mathrm{CH}_{3}\right)_{2}\right] ; 1.83-2.24$ (m, 4H, H-1", H-2'); 3.01 (m, 2H, H-2"); 3.72 (m, 2H, H-5'a, H-4'); 4.01 (m, 1H, H-5'b); 4.16 (m, 1H, H-1'); 4.39 (dt, 1H, ${ }^{3} J_{3^{\prime}, 2^{\prime} \mathrm{b}} 5.0 \mathrm{~Hz},{ }^{3} J_{3^{\prime}, 2^{\prime} \mathrm{a}} 8.0 \mathrm{~Hz}$, $\left.{ }^{3} J_{3^{\prime}, 4^{\prime}} 8.0 \mathrm{~Hz}, \mathrm{H}-3^{\prime}\right) ; 6.81$ (d, 1H, $\left.{ }^{3} J_{3,4} 7.0 \mathrm{~Hz}, \mathrm{H}-3\right) ; 7.38$ (m, 1H, H-7); 7.53 (m, 1H, H-8); 7.83 $\left(\mathrm{d}, 1 \mathrm{H},{ }^{3} J_{6,7} 8.2 \mathrm{~Hz}, \mathrm{H}-6\right) ; 7.97$ (d, $\left.1 \mathrm{H},{ }^{3} J_{8,9} 8.2 \mathrm{~Hz}, \mathrm{H}-9\right) ; 8.60$ (d, 1H, H-4). ${ }^{13} \mathrm{C}$ NMR (62.9 $\left.\mathrm{MHz}, \mathrm{CDCl}_{3}\right) \delta 12.5,12.9,13.3,13.5\left[4 \mathrm{CH}\left(\mathrm{CH}_{3}\right)_{2}\right] ; 16.9,17.0,17.1,17.2,17.3,17.4,17.5$ (2) [4 $\mathrm{CH}\left(\mathrm{CH}_{3}\right)_{2}$ ]; 33.5 (C-1"); 35.1 (C-2"); 40.3 (C-2'); 63.8 (C-5'); 73.5 (C-3'); 76.8 (C-1'); 85.9 (C-4'); 107.6 (C-3); 110.3 (C-6); 120.3 (C-9); 121.7 (C-7); 126.2 (C-8); 126.9 (C-5a); 132.3 (C4); 144.0 (C-9a); 150.7 (C-10a); 169.1 (C-2). ESI-MS (-): $m / z=554$ [M-H] $]^{-}$Anal. Calcd for $\mathrm{C}_{29} \mathrm{H}_{45} \mathrm{~N}_{3} \mathrm{O}_{4} \mathrm{Si}_{2}$ (555.86): C, 62.66; H, 8.16; N, 7.56. Found: C, 62.89; H, 8.29; N, 7.37.

\section{Acknowledgements}

This work has been supported by the DFG Research Training Group 1213 "New Methods for Sustainability in Catalysis and Technique".

\section{References}

1. Bobek, M. ; Farkas, J. Collect. Czech. Chem. Commun. 1969, 34, 1684.

2. Sato, T.; Noyori, R. Bull. Chem. Soc. Jpn. 1983, 56, 2700.

3. Bisht, S. S.; Jaiswal, N.; Sharma, A.; Fatima, S.; Sharma, R.; Rahuja, N.; Srivastava, A. K.; Bajpai, V.; Kumar, B.; Tripathi, R. P. Carbohydr. Res. 2011, 346, 1191.

4. Haraguchi, K.; Delaney, M. O.; Wiederholt, C. J.; Sambandam, A.; Hantosi, Z.; Greenberg, M. M. J. Am. Chem. Soc. 2002, 124, 3263.

5. Ciba, J. Takeshima, S.; Mishima, K.; Maeda, H.; Nanai, Y.; Mizuno, K.; Inouye, M. Chem. Eur. J. 2007, 13, 8124.

6. Liu, H.; Gao, J. Kool, E. T. J. Am. Chem. Soc. 2005, 127, 1396.

7. Lee, A. H. F.; Kool, E. T. J. Am. Chem. Soc. 2005, 127, 3332.

8. Lee, A. H. F.; Kool, E. T. J. Org. Chem. 2005, 70, 132.

9. Liu, H.; Gao, J. Kool, E. T. J. Org. Chem. 2005, 70, 639.

10. Krueger, A. T.; Kool, E. T. J. Am. Chem. Soc. 2008, 130, 3989.

11. Otero Martinez, H. Reinke, H. Michalik, D.; Vogel, C. Synthesis 2009, 1834.

12. Waechtler, H.; Pena Fuentes, D.; Michalik, D.; Koeckerling, M.; Villinger, A.; Kragl, U. Cedeno, Q. A.; Vogel, C. Synthesis 2011, 3099.

13. Otero, I.; Feist, H.; Herrera, L.; Michalik, M.; Quincoces, J.; Peseke, K. Carbohydr. Res. 2005, 340, 547. 
14. Gewald, K.; Schinke, E.; Boettcher, H. Chem.Ber. 1966, 99, 94.

15. Taylor, E. C.; Berger, J. G. J. Org. Chem. 1967, 32, 2376.

16. Brennan, C. M.; Johnson, C. D ; McDonnell, P. D. J. Chem. Soc., Perkin Trans. 2 1989, 957.

17. Adlington, R. M.; Baldwin, J. E.; Catterick, D.; Pritchard, G. J. J. Chem. Soc., Perkin Trans. 1 1999, 855.

18. Adlington, R. M.; Baldwin, J. E.; Catterick, D.; Pritchard, G. J.; Tang, L. T. J. Chem. Soc., Perkin Trans. 1 2000, 2311.

19. Logue, M. W.; Sarangan, S. Nucleos Nucleot 1982, 1, 89.

20. Otero, I.; Feist, H.; Herrera, L.; Michalik, M.; Quincoces, J. Peseke, K. Aust. J. Chem. 2005, $58,104$.

21. Otero, I.; Feist, H.; Herrera, L.; Michalik, D.; Michalik, M.; Quincoces, J. Peseke, K. Z. Naturforsch. 2005, 60b, 1175.

22. Perrin, D. D.; Armarego, W. L. F., Purification of Laboratory Chemicals, $3^{\text {rd }}$ Ed.; Pergamon Press: Oxford, 1988. 